\title{
High Temperature Expansions and Dynamical Systems
}

\author{
J.Bricmont* \\ UCL, Physique Théorique, B-1348, Louvain-la-Neuve, Belgium \\ bricmont@fyma.ucl.ac.be \\ A.Kupiainen ${ }^{\dagger}$ \\ Helsinki University, Department of Mathematics, \\ Helsinki 00014, Finland \\ ajkupiai@cc.helsinki.fi
}

\begin{abstract}
We develop a resummed high-temperature expansion for lattice spin systems with long range interactions, in models where the free energy is not, in general, analytic. We establish uniqueness of the Gibbs state and exponential decay of the correlation functions. Then, we apply this expansion to the Perron-Frobenius operator of weakly coupled map lattices.
\end{abstract}

\section{Introduction.}

The theory of Gibbs states was originally developed for the mathematical analysis of equilibrium statistical mechanics. An interesting application of the theory was found by Sinai, Ruelle and Bowen in the 70's 41, 42, 38, 1] who applied it to the ergodic theory of uniformly hyperbolic dynamical systems. While this so called thermodynamic formalism has been very successful in ergodic theory, the Gibbs states that describe the statistics of such dynamical systems are quite simple from the point of view of statistical mechanics: they describe one dimensional spin systems with spins taking values in a finite set and interacting with exponentially decaying potentials. In particular, phase transitions, i.e. the coexistence of several Gibbs states for the same interaction, which are of major interest in statistical mechanics, are absent in such systems.

More recently, it has been realized that certain infinite dimensional dynamical systems possess attracting sets that are extensive in a suitable volume. This is believed to be the case for many classes of nonlinear parabolic partial differential equations on

*Supported by EC grants SC1-CT91-0695 and CHRX-CT93-0411

†Supported by NSF grant DMS-9205296 and EC grant CHRX-CT93-0411 
some spatial domain: the dimension of the attracting set (or a bound for it) increases to infinity as the domain becomes unbounded [43]. Discrete time dynamical systems, such as coupled maps, were introduced to model these phenomena [29]. Bunimovich and Sinai [5] showed that these systems give rise to a thermodynamic formalism for spin systems on a lattice of more than one dimension. Because of this last feature, the possibility of phase transitions is at least open. However, most rigorous analyses have so far been limited to weakly coupled maps which corresponds to "high temperature" in statistical mechanics. But, even in that regime, coupled maps give rise to interactions of a rather peculiar kind, and proving the absence of phase transitions for the latter is not completely trivial.

In statistical mechanics, the common wisdom is that "high temperature" leads to uniqueness and decay of correlations (mixing for lattice translations). Mathematically, one introduces a suitable Banach space of "interactions" parametrizing the Gibbs states and high temperature means small norm. For small norm the interaction uniquely determines the Gibbs state [10, 11]. However, the question of a suitable norm turns out to be subtle. One can distinguish between three properties: uniqueness of the Gibbs state, exponential mixing and analyticity (in the potentials) of the correlation functions. In the last case one has a complete control of the Gibbs state in terms of a convergent expansion, the high temperature expansion. In the other two cases, less detailed information is available.

As we shall recall in Section 2, there exist Banach spaces of interactions where exponential mixing holds, but analyticity does not hold, in general. It turns out that the interactions corresponding, in the thermodynamic formalism, to coupled maps belong to such "pathological" Banach spaces of interactions.

The purpose of this paper is twofold. First we wish to give a unified treatment of the high temperature states discussed above. We show how all the previous results (e.g. the uniqueness results of Dobrushin [10, 11]) and also the generalizations needed for dynamical systems can be obtained by a simple resummation of a high temperature expansion based on an idea of von Dreifus, Klein and Perez [17 used for disordered systems (such a resummation was used already by Fisher [20]). The resummation uses in an essential way the fact that the interactions are real and thus is not in conflict with the lack of analyticity.

Then we apply these results to the study of the ergodic theory of coupled $C^{1+\delta}$ circle maps. We rederive, generalize and complete the previous studies of such maps. We believe this is necessary due to some confusion and incorrect results in the litterature (see Section 4). Our approach is slightly novel since we derive the thermodynamic formalism without introducing Markov partitions and symbolic dynamics (which however exist in the models we consider). One motivation for this is that systems where one expects phase transitions (based on numerical and theoretical evidence [37, 33]) will, most probably, not have useful Markov partitions. In our formalism such systems (e.g. coupled bounded variation interval maps) nevertheless give rise to a thermodynamic formalism, but with potentials whose thermodynamic limit we are presently unable to control.

The paper is organized as follows. Section 2 contains a review of the theory of Gibbs states and of the various spaces of potentials. We also formulate and prove in Section 
2 our main statistical mechanics theorem. This Section is completely independent of the dynamical system part of the paper. Section 3 defines coupled map lattices and states the main theorem, whose proof can be found in Section 4. We have tried to be rather self-contained in the statistical mechanics hoping the paper to be accessible to the dynamical systems community.

\section{Resummed high-temperature expansions.}

\subsection{Lattice systems}

We consider a lattice spin system: to each $i \in \mathbf{Z}^{d}$ we $\operatorname{assign} \Omega_{i}$, a copy of a finite set $\Omega_{0}$ or more generally a compact metric space; we stick to the former, but the generalization to the latter case is straightforward. Given $X \subset \mathbf{Z}^{d}$, a spin configuration in $X$, denoted by $s_{X}$, is an element $s_{X} \in \Omega_{X} \equiv \times_{i \in X} \Omega_{i}$. An interaction is defined by a family $\Phi=\left(\Phi_{X}\right)$ of (continuous) functions indexed by finite subsets $X$ of $\mathbf{Z}^{d}$ :

$$
\Phi_{X}: \Omega_{X} \rightarrow \mathbf{R}
$$

We let $\left\|\Phi_{X}\right\|$ denote the sup norm of $\Phi_{X}$. We consider translation invariant $\Phi^{\prime} s$ : $\Phi_{X+i}=\tau_{i} \Phi_{X}$ where $\tau_{i}, i \in \mathbf{Z}^{d}$ is the natural $\mathbf{Z}^{d}$ action on functions defined on $\Omega_{X}$ to functions defined on $\Omega_{X+i}$. Given $\Lambda \subset \mathbf{Z}^{d},|\Lambda|<\infty$, and a configuration $s_{\Lambda^{c}}^{\prime}=\left(s_{i}^{\prime}\right)_{i \in \Lambda^{c}}$ in $\Omega_{\Lambda^{c}}$, the Hamiltonian in $\Lambda$ (with boundary conditions $s_{\Lambda^{c}}^{\prime}$ ) is defined as

$$
\mathcal{H}\left(s_{\Lambda} \mid s_{\Lambda^{c}}^{\prime}\right)=-\sum_{X \cap \Lambda \neq \emptyset} \Phi_{X}\left(s_{X \cap \Lambda} \vee s_{X \cap \Lambda^{c}}^{\prime}\right)
$$

where, if $s_{X} \in \Omega_{X}, s_{Y}^{\prime} \in \Omega_{Y}$, for $X \cap Y=\emptyset, s_{X} \vee s_{Y}^{\prime}$ is the obvious configuration in $\Omega_{X \cup Y}$. The associated (finite volume) Gibbs measure is a probability distribution on $\Omega_{\Lambda}$ :

$$
\nu\left(s_{\Lambda} \mid s_{\Lambda^{c}}^{\prime}\right)=Z^{-1}\left(\Lambda \mid s_{\Lambda^{c}}^{\prime}\right) \exp \left(-\mathcal{H}\left(s_{\Lambda} \mid s_{\Lambda^{c}}^{\prime}\right)\right)
$$

(we put a minus sign in (2) for convenience), with

$$
Z\left(\Lambda \mid s_{\Lambda^{c}}^{\prime}\right)=\sum_{s_{\Lambda}} \exp \left(-\mathcal{H}\left(s_{\Lambda} \mid s_{\Lambda^{c}}^{\prime}\right)\right)
$$

See e.g. [38, 40, 19] for more details on the theory of Gibbs states.

\subsection{Finite range interactions}

Suppose first that $\Phi_{X}$ is of finite range, i.e., for some $R<\infty, \Phi_{X}=0$ if the diameter of $X, d(X)>R$ (we put on $\mathbf{Z}^{d}$ the metric $\left.|i|=\max _{\alpha}\left|i_{\alpha}\right|\right)$. Then, if $\sup _{X}\left\|\Phi_{X}\right\|$ is small enough, a convergent high-temperature expansion (so-called polymer expansion) yields the following results (see [21, 4, 32, 40]): 
1) The Gibbs state is unique i.e., the finite-volume Gibbs measures (3) converge, as $\Lambda \uparrow \mathbf{Z}^{d}$, independently of the boundary conditions to a unique measure on $\Omega_{\mathbf{Z}^{d}}$ satisfying certain consistency conditions (the DLR-equations).

2) The correlation functions in that Gibbs measure decay exponentially (see (12) below).

3) The free energy $F(\Phi)$ defined as $\lim _{\Lambda \rightarrow \infty}|\Lambda|^{-1} \log Z\left(\Lambda \mid s_{\Lambda^{c}}^{\prime}\right)$ and the correlation functions are analytic: Given finite subsets $X_{1}, \ldots, X_{n}$ and $\lambda \in \mathbf{C}^{n}$, define $\Phi^{\lambda}$ by $\Phi_{Y}^{\lambda}=\lambda_{\alpha} \Phi_{Y}$ for each $Y=X_{\alpha}+j$, for some $j \in \mathbf{Z}^{d}$ and some $\alpha=1, \cdots, n$ and $\Phi_{Y}^{\lambda}=\Phi_{Y}$ for the other $Y^{\prime}$ 's. Then, $F\left(\Phi^{\lambda}\right)$ is analytic in the polydisc $\left|\lambda_{\alpha}\right| \leq \epsilon$, for $\epsilon$ small enough.

The smallness of $\left\|\Phi_{X}\right\|$ is however not a necessary condition for uniqueness and analyticity. For example, one dimensional finite range systems always satisfy all three conditions above. Dobrushin-Shlosman [15, 16] gave several equivalent conditions to be satisfied by the finite volume distributions (3) that guarantee that the Gibbs state is unique and that analyticity and exponential decay of correlations hold (they call these properties "complete analyticity"). These results were later rederived by Olivieri and Picco [34, 35] using expansion methods.

To formulate one of these conditions, used in [34, 35], it is convenient to cover $\mathbf{Z}^{d}$ by disjoint cubes of side $L$, called $L$-cubes. We fix such a covering. We choose $L$ sufficiently large, and, in particular $L>R$, where $R$ is the range of $\Phi^{0}$. Then the condition becomes: There exists a function $f: \mathbf{N} \rightarrow \mathbf{R}$ such that

$$
\left|\frac{Z\left(\Lambda \mid s^{x y}\right) Z(\Lambda \mid s)}{Z\left(\Lambda \mid s^{x}\right) Z\left(\Lambda \mid s^{y}\right)}-1\right| \leq f(|x-y|)
$$

and

$$
\lim _{n \rightarrow \infty} n^{2(d-1)} f(n)=0 .
$$

Here, $\Lambda$ is an arbitrary union of $L$-cubes and $s$ an arbitrary configuration in $\Omega_{\Lambda^{c}} ; s_{z}^{A}=s_{z}$ for $z \notin A$, where $A=x, y$ or $x y$. (Actually, it is enough to check (5) for $\Lambda$ 's being subsets of a sufficiently large volume. Then, the fact that (5) holds for larger volumes follows from (13) below.)

\subsection{Infinite range interactions}

Many of these results extend to infinite range interactions but one has to carefully distinguish between different Banach spaces of interactions in which different results are valid. Without trying to be exhaustive, one has basically the following results: (see 40] for a review)

1) If

$$
\|\Phi\|_{1}=\sum_{0 \in X}|X|\left\|\Phi_{X}\right\|
$$

is small enough, then the Gibbs state is unique [10, 11, 42] 
2) If, for some $\gamma>0$,

$$
\|\Phi\|_{2}=\sum_{0 \in X} e^{\gamma d(X)}\left\|\Phi_{X}\right\|
$$

is small enough, then the Gibbs state is unique and its correlation functions decay exponentially [23].

3) If, for some $\gamma>0$,

$$
\|\Phi\|_{3}=\sum_{0 \in X} e^{\gamma|X|}\left\|\Phi_{X}\right\|
$$

is small enough, then the Gibbs state is unique and its correlation functions are analytic [26].

The reasons for these different norms are as follows: usually, correlation functions do not decay faster than the interactions, so that something like (8) is needed to get exponential decay.

As for analyticity, Dobrushin and Martirosyan [13 have shown, by explicit counterexamples, that analyticity will not hold, (at least not uniformly in the volume, see [19], p.958), in a neighbourhood of zero, in any space larger than the one defined by (9): Let

$$
\|\Phi\|_{h}=\sum_{0 \in X} h(|X|)\left\|\Phi_{X}\right\| .
$$

Then, if $\lim _{n \rightarrow \infty} h(n) e^{-\gamma n}=0, \forall \gamma>0$, there exist (complex) interactions with arbitrarily small $\|\Phi\|_{h}$ such that the corresponding partition function vanishes for a sequence of cubes $\Lambda_{n} \rightarrow \infty$. (see [18], p.971 for a simple such counterexample).

From the point of view of polymer expansions, the norm (9) is quite natural, as was remarked by Brydges (四 p.141): the exponential weight in (9), depending on the size of $X$, is needed to control the sum over small polymers lying inside a big one.

However, this norm is stronger than (7) and, sometimes, than (8): if the lattice dimension $d$ is larger than one, and if $X$ is, e.g., a large cube, $|X|>>d(X)$. So, for some $\Phi$ 's, uniqueness and exponential decay hold but analyticity does not, and it seems that the first two properties cannot be proven using convergent polymer expansions (the existing proofs use Dobrushin's methods). The problem is that these expansions tend, when they converge, to yield analyticity almost automatically.

The reader should not be misled by the fact that standard high-temperature expansions for spin $\frac{1}{2}$ systems [21, 26, 9] seem to show analyticity for interactions that are small in a weaker norm than (9). Indeed, in these papers, the interactions are written in a special representation ("spin" or "gas" language) and the norm used for the interactions is quite different from the sup norm. This slightly confusing point is very nicely clarified in [18]. 


\subsection{The main result}

The analyticity results of Dobrushin and Shlosman were extended, for the analyticity part, by Dobrushin and Martirosyan [12] to infinite range interactions of the form

$$
\Phi=\Phi^{0}+\Phi^{1}
$$

where $\Phi^{0}$ has finite range and is completely analytic, while $\left\|\Phi^{1}\right\|_{3}$ is small (actually, Dobrushin and Martirosyan discuss the $\Phi^{0}$ part in terms of specifications instead of interactions; this could be done here too). In the dynamical systems problem of Section 3 we will encounter an interaction of even more general type, namely of the form (11), but with $\left\|\Phi^{1}\right\|_{2}$ (instead of $\left.\left\|\Phi^{1}\right\|_{3}\right)$ small. Our main result is

Theorem 1 Let $\Phi=\Phi^{0}+\Phi^{1}$, where $\Phi^{0}$ is completely analytic. Then, there exist $\epsilon>0$, $m>0, C<\infty$ such that, if $\left\|\Phi^{1}\right\|_{2} \leq \epsilon$, there is a unique Gibbs state $\mu$ for $\Phi$ and the correlation functions satisfy, for all $F, G$, with $F: \Omega_{A} \rightarrow \mathbf{R}, G: \Omega_{B} \rightarrow \mathbf{R}$, where $A, B \subset \mathbf{Z}^{d}$ are finite:

$$
|\langle F G\rangle-\langle F\rangle\langle G\rangle| \leq C\|F\|\|G\| e^{-m d(A, B)}
$$

where $d(A, B)$ is the distance between the sets $A$ and $B$ and $\langle F\rangle=\int F d \mu$.

Remark 1. We shall discuss various extensions and variations of this result after giving the proof in Section 2.5.

Remark 2. Our proof is based on a high-temperature expansion instead of Dobrushin's method. We shall resum the expansion and use then, in an essential way, the fact that $\Phi_{X}$ is real, so that our proof applies even when analyticity does not hold. It is inspired by a recent work of von Dreifus, Klein and Perez [17] who developed a high-temperature expansion for disordered systems. Their problem was similar to ours: due to Griffiths' singularities [22], analyticity does not hold, in general, for disordered systems, while we have to circumvent the possibility of Dobrushin-Martirosyan singularities [13. Actually, a simple version of our method appears already in Fisher's upper bound on the twopoint function for the Ising model [20]. On the other hand, in a recent paper, Jiang and Mazel 28] consider real interactions similar to ours, and develop a convergent hightemperature expansion which yields uniqueness and exponential decay of the correlation functions, but for systems defined on a two-dimensional lattice (their method crucially depends on the fact that in two dimensions boundaries of volumes are proportional to their diameters).

\subsection{The proof}

Before starting with the proof, we will use another representation for the partition function of $\Phi^{0}$ derived from $(5,6)$ by Olivieri and Pico. They show that if $(5,6)$ holds then the interaction $\Phi^{0}$ "has a cluster (or polymer) expansion". Precisely, this means 
that the partition function admits the following representation. If $\Lambda$ is a union of disjoint $L$-cubes, and $s \in \Omega_{\Lambda^{c}}$,

$$
Z^{0}(\Lambda \mid s)=e^{f|\Lambda|} \prod_{\alpha} W\left(s_{\Lambda_{\alpha}^{c}}\right) \exp \sum_{Y} \phi_{Y}\left(s_{Y \cap \Lambda^{c}}\right)
$$

where $Z^{0}$ refers to the partition function with interaction $\Phi^{0}$, the product runs over connected components of $\Lambda^{c}=\cup_{\alpha} \Lambda_{\alpha}^{c}$ and the sum runs over connected sets of $L$-cubes $Y$, so that $Y \cap \Lambda \neq \emptyset$ (and $\phi_{Y}$ is a constant if $\left.Y \cap \Lambda^{c}=\emptyset\right)$. We define a subset $X \subset \mathbf{Z}^{d}$ to be connected if $\forall i, j \in X$ there exists a path $i(1), \cdots, i(\ell)$ with $i(1)=i, i(\ell)=j, i(k) \in$ $X,|i(k)-i(k+1)|=1, \forall k$, where $|i|=\max _{\alpha}\left|i_{\alpha}\right| . W \geq 0$ depends on $s_{i}$, for $i \in \Lambda_{\alpha}^{c}$, such that $d(i, \Lambda) \leq R$. We have the following bound: for any $\epsilon>0$, there exist $L<\infty$, $\gamma>0$ such that, for the expansion defined with $L$-cubes,

$$
\sum_{0 \in Y} \exp \left(\frac{\gamma d(Y)}{L}\right)\left\|\phi_{Y}\right\| \leq \epsilon
$$

(there is no loss of generality in assuming that $\gamma$ here is the same as in (8)).

Remark. Our condition (5) is Condition A in [34 (together with (3.7) in $d=2$ ), or Condition $\mathrm{C}$ in [35] (see (2.65)). The representation (13) follows from (2.53), (2.56), (2.60) in [35], or, more precisely, from the extension of (2.53) to arbitrary boundary conditions. In [34, 35] volumes of various shapes are used, but one can always regroup terms and index them by $L$-cubes, as was done here for simplicity of notations. The reader may notice that (13) is what we get at high temperatures, the only difference being that a site is replaced here by an $L$-cube: $f$ is the bulk free energy, the product over the $W$ 's gathers all the boundary terms, and the sum over $\phi_{Y}$ is the usual cluster expansion. The constant $L$ is, in effect, of the order of the correlation length of the system. Note also that, if $\Phi^{0}$ is a finite range one-dimensional interaction (i.e. it couples spins only along lines parallel to a lattice axis), then the transfer matrix formalism implies that (5) holds. In our application to coupled maps, $\Phi^{0}$ will be exactly of that form (see Proposition 3 in Section 4).

Remark In the proofs, we shall denote by $C$ or $c$ a constant that may vary from place to place.

Proof of Theorem 1. We shall prove (12) where the expectation will be taken with respect to a finite volume Gibbs state with open boundary conditions (to simplify notations), i.e. the sum in (2) is restricted to $X \subset \Lambda$. The corresponding expectation is written $\langle\cdot\rangle_{\Lambda}$. Our bounds are uniform in $\Lambda$. We can then study the limit $\Lambda \uparrow \mathbf{Z}^{d}$, and prove (12) in that limit. The uniqueness of the Gibbs state can be shown in a similar way, and will be discussed at the end of the proof.

First, we write the LHS of (12) using duplicate variables:

$$
=\sum_{s_{\Lambda}^{i}, i=1,2}\left(F\left(s_{\Lambda}^{1}\right)-F\left(s_{\Lambda}^{2}\right)\right)\left(G\left(s_{\Lambda}^{1}\right)-G\left(s_{\Lambda}^{2}\right)\right) \nu\left(s_{\Lambda}^{1}\right) \nu\left(s_{\Lambda}^{2}\right)
$$




$$
=Z(\Lambda)^{-2} \sum_{s_{\Lambda}^{i}, i=1,2} \tilde{F} \tilde{G} \exp \left(-\mathcal{H}\left(s_{\Lambda}^{1}\right)-\mathcal{H}\left(s_{\Lambda}^{2}\right)\right)
$$

where $\tilde{F}=F\left(s_{\Lambda}^{1}\right)-F\left(s_{\Lambda}^{2}\right), \tilde{G}=G\left(s_{\Lambda}^{1}\right)-G\left(s_{\Lambda}^{2}\right)$.

We may replace $\Phi_{X}^{1}$ by $\Phi_{X}^{1}-\inf _{s_{X}} \Phi_{X}^{1}$, by adding a constant to the Hamiltonian. Thus, we may, without loss of generality, assume that $\Phi_{X}^{1} \geq 0$ for all $X$, and that $\Phi^{1}$ still satisfies $\left\|\Phi^{1}\right\|_{2} \leq 2 \epsilon$. Now, we perform a usual high-temperature expansion on the $\Phi^{1}$ part of $\mathcal{H}$ :

$$
\exp \left(\sum_{X, i=1,2} \Phi_{X}^{1}\left(s_{X}^{i}\right)\right)=\sum_{\mathcal{X}} \prod_{X \in \mathcal{X}} f_{X}
$$

where the sum runs over sets $\mathcal{X}$ of subsets of $\Lambda$, and

$$
f_{X}=\exp \left(\Phi_{X}^{1}\left(s_{X}^{1}\right)+\Phi_{X}^{1}\left(s_{X}^{2}\right)\right)-1
$$

satisfies:

$$
\begin{gathered}
0 \leq f_{X} \\
\sum_{0 \in X} e^{\gamma d(X)}\left\|f_{X}\right\| \leq C \epsilon
\end{gathered}
$$

(Use (8) and $\left.\left\|\Phi^{1}\right\|_{2} \leq \epsilon\right)$. Insert (16) in (15) and, for each term in (16), define $V=$ $V(\mathcal{X})=\underline{A} \cup \underline{B} \cup \underline{\mathcal{X}}$, where $\underline{\mathcal{X}}=\cup_{X \in \mathcal{X}} \underline{X}$ and for any $X \subset \mathbf{Z}^{d}, \underline{X}$ is the set of $L$-cubes intersected by $X$. We have

$$
(15)=Z(\Lambda)^{-2} \sum_{\mathcal{X}} \sum_{s_{V}^{i}, i=1,2} \tilde{F} \tilde{G} \prod_{X \in \mathcal{X}} f_{X} \exp \left(-\mathcal{H}_{V}^{0}\right) \prod_{i=1,2} Z^{0}\left(\Lambda \backslash V \mid s_{V}^{i}\right)
$$

where $\mathcal{H}_{V}^{0}=-\sum_{X \subset V}\left(\Phi_{X}^{0}\left(s_{X}^{1}\right)+\Phi_{X}^{0}\left(s_{X}^{2}\right)\right)$, and $Z^{0}\left(\Lambda \backslash V \mid s_{V}^{i}\right)$ is the partition function with interaction $\Phi^{0}, s_{V}^{i}$ boundary condition in $V$, and open boundary conditions in $\Lambda^{c}$. Now, we use (13) for $Z^{0}\left(\Lambda \backslash V \mid s_{V}^{i}\right)$, and we define $\Psi_{Y}\left(s_{Y \cap V}\right)$ by:

$$
\phi_{Y}\left(s_{Y \cap V}\right)=\Psi_{Y}\left(s_{Y \cap V}\right)+\bar{\phi}_{Y}
$$

where

$$
\bar{\phi}_{Y}=\min _{s_{Y \cap V}} \phi_{Y}\left(s_{Y \cap V}\right)
$$

so that $\Psi_{Y} \geq 0$. Then, we perform a high-temperature expansion of $\Psi_{Y}$ :

$$
\exp \left(\sum_{Y, i=1,2} \Psi_{Y}\left(s_{Y \cap V}^{i}\right)\right)=\sum_{\mathcal{Y}} \prod_{Y \in \mathcal{Y}} g_{Y}
$$

where $Y \cap(\Lambda \backslash V) \neq \emptyset$ and $\mathcal{Y}$ is a set of such $Y^{\prime}$ 's; $g_{Y}=\exp \left(\Psi_{Y}\left(s_{Y \cap V}^{1}\right)+\Psi_{Y}\left(s_{Y \cap V}^{2}\right)\right)-1$ satisfies:

$$
0 \leq g_{Y}
$$




$$
\sum_{0 \in Y} \exp \left(\frac{\gamma d(Y)}{L}\right)\left\|g_{Y}\right\| \leq C \epsilon
$$

(using (14)). Finally, we insert the result in (20). We get

$$
(20)=Z(\Lambda)^{-2} \sum_{\mathcal{X}, \mathcal{Y}} \sum_{s_{V}^{i}, i=1,2} \tilde{F} \tilde{G} \prod_{X \in \mathcal{X}} f_{X} \prod_{Y \in \mathcal{Y}} g_{Y} \exp \left(-\mathcal{H}_{V}^{0}\right) \mathcal{W} \exp \left(2 \sum_{Y} \bar{\phi}(Y)\right)
$$

where $\mathcal{W}=e^{2 f|\Lambda| V \mid} \prod_{\alpha, i=1,2} W\left(s_{V_{\alpha}}^{i}\right)\left(V_{\alpha}\right.$ are the connected components of $\left.V\right)$, and the sum over $Y$ runs over $Y \cap(\Lambda \backslash V) \neq \emptyset$.

Now comes the main observation: for each term of (26), decompose $V \cup\{Y \mid Y \in \mathcal{Y}\}$ into connected components. If $\underline{\mathrm{A}}$ and $\underline{\mathrm{B}}$ are in different components, then the corresponding term in (26) vanishes. Indeed, because of the factor $\tilde{F} \tilde{G}$, the summand is then odd under the interchange of $s_{i}^{1}$ and $s_{i}^{2}$ for all $i$ in the component containing $\underline{\mathrm{A}}$ (or B). Here, we use the fact that, since $L>R, R$ being the range of $\Phi_{0}$, every $X \subset V$ contributing to the sum defining $\mathcal{H}_{V}^{0}$, belongs to a single connected component $V_{\alpha}$.

So, for each non-vanishing term in (26), we can choose a "connected path" $P=$ $\left(Z_{i}\right)_{i=1}^{n}$ where $Z_{1}=\underline{A}, Z_{n}=\underline{B}$, each $Z_{i}$ for $i \neq 1, n$ is either an $\underline{X}$ or a $Y$ and $Z_{i+1}$ is adjacent to $Z_{i}$ (i.e. $d\left(Z_{i}, Z_{i+1}\right) \leq 1$ ), $\forall i=1, \cdots, n-1$. So we get a bound on (26):

$$
\begin{aligned}
& |(26)| \leq Z(\Lambda)^{-2} 4\|F\|\|G\| \sum_{P} \prod_{\underline{X} \in P}\left\|f_{X}\right\| \prod_{Y \in P}\left\|g_{Y}\right\| \sum_{\mathcal{X}, \mathcal{Y}}{ }^{P} \sum_{s_{V}^{i}} \prod_{X \in \mathcal{X}, \underline{x} \notin P} f_{X} \prod_{X \in \mathcal{X}, \underline{X} \in P}\left(1+f_{X}\right) \\
& \prod_{Y \in \mathcal{Y} \backslash P} g_{Y} \prod_{Y \in P}\left(1+g_{Y}\right) \exp \left(-\mathcal{H}_{V}^{0}\right) \mathcal{W} \exp \left(2 \sum_{Y} \bar{\phi}(Y)\right)
\end{aligned}
$$

where we used the positivity of $f_{X}, g_{Y}, \mathcal{W}$ (in particular, in order to insert the products over $\left.1+f_{X}, 1+g_{Y}\right)$, we bounded $\tilde{F}, \tilde{G}$ by $4\|F\|\|G\|$, and we wrote $\sum^{P}$ to denote the sum over the pairs $(\mathcal{X}, \mathcal{Y})$ for which the chosen path is $P$. Now, observe that, by resumming the expansions, the sum over $\mathcal{X}, \mathcal{Y}, s_{V}^{i}$ is less than $Z(\Lambda)^{2}$ : we get an upper bound on $\sum_{\mathcal{X}, \mathcal{Y}}^{P}$ by resumming first over all $\mathcal{Y}$ that are sets of $Y$ 's intersecting $\Lambda \backslash V$, with $Y \notin P$ and then over all $\mathcal{X}$ that are sets of $X$ 's in $\Lambda$, with $\underline{X} \notin P$ (since each term in (27) is positive). This upper bound is equal to $Z(\Lambda)^{2}$. So, we finally get

$$
(27) \leq 4\|F\|\|G\| \sum_{P} \prod_{\underline{X} \in P}\left\|f_{X}\right\| \prod_{Y \in P}\left\|g_{Y}\right\|
$$

Now, to get (12), we have to bound the sum over $P$. First use

$$
d(A, B) \leq c L\left(1+\sum_{\underline{X} \in P} d(X)\right)+\sum_{Y \in P} d(Y)
$$

for some $c<\infty$, where the factor $c L$ enters because we consider $Z_{1}=\underline{A}, Z_{n}=\underline{B}$, or $Z_{i}=$ $\underline{X}$, which are unions of $L$-cubes. We choose $m=\frac{\gamma}{2 c L}$, and use (29) to bound $e^{m d(A, B)}$. Finally, we have to control the sum over $P$ in (28). Using (29) and $(15,20,26,27,28)$, we bound

$$
e^{m d(A, B)}\left|\langle F G\rangle_{\Lambda}-\langle F\rangle_{\Lambda}\langle G\rangle_{\Lambda}\right| \leq 2\|F\|\|G\| \sum_{n=2}^{\infty} \sum_{\left(Z_{i}\right)_{2}^{n-1}} \prod_{i=2}^{n-1} h\left(Z_{i}\right)
$$


where each $Z_{i}$ is either a $\underline{X}$ or a $Y, h(Z)=\exp \left(\frac{\gamma d(X)}{2}\right)\left\|f_{X}\right\|$ or $\exp \left(\frac{\gamma d(Y)}{2 c L}\right)\left\|g_{Y}\right\|$ and each $Z_{i}$ is adjcacent to $Z_{i-1}$. We can bound the sum over $\left(Z_{i}\right)$ by the (ordered) product

$$
\prod_{i=2}^{n-1}\left(\sum_{Z_{i}}^{i-1} h\left(Z_{i}\right)\right)
$$

where $\sum^{i-1}$ means that $Z_{i}$ is adjacent to $Z_{i-1}$. Now,

$$
\sum_{Z_{i}}^{i-1} h\left(Z_{i}\right) \leq C|X|\left(\sum_{0 \in Z} h(Z)\right)
$$

if $Z_{i-1}$ is an $\underline{X}$, or

$$
\sum_{Z_{i}}^{i-1} h\left(Z_{i}\right) \leq C|Y|\left(\sum_{0 \in Z} h(Z)\right)
$$

if $Z_{i-1}$ is a $Y$, since the number of translates of $Z_{i}, 0 \in Z_{i}$ that are adjacent to $Z_{i-1}$ (with $Z_{i-1}=\underline{X}$ or $Y$ ) is bounded by a constant times $|X|$ or $|Y|$, respectively. We then use the bounds

$$
\sum_{0 \in \underline{X}}|X| \exp \left(\frac{\gamma d(X)}{2}\right)\left\|f_{X}\right\| \leq C L^{d} \epsilon,
$$

and

$$
\sum_{0 \in Y}|Y| \exp \left(\frac{\gamma d(Y)}{2 c L}\right)\left\|g_{Y}\right\| \leq C L^{d} \epsilon,
$$

which follow from $(19,25)$ (in (30), we use the fact that the sum over $0 \in \underline{X}$ is bounded by $C L^{d}$ times the sum over $0 \in X$, and $|X| \leq C d(X)^{d}$ while in (31), we use $|Y| \leq$ $\left.C d(Y)^{d} \leq C(2 c L)^{d}\left(\frac{d(Y)}{2 c L}\right)^{d}\right)$. Finally, for $\epsilon$ small enough, we bound (28) by a convergent geometric series:

$$
e^{m d(A, B)}\left|\langle F G\rangle_{\Lambda}-\langle F\rangle_{\Lambda}\langle G\rangle_{\Lambda}\right| \leq 2\|F\|\|G\| \sum_{n=0}^{\infty}\left(C L^{d} \epsilon\right)^{n}
$$

To prove the existence of the $\Lambda \uparrow \mathbf{Z}^{d}$ limit (in the sense of finite subsets ordered by inclusion) and the uniqueness of the Gibbs state, consider $F$ as in (12) and two boundary conditions $s \in \Omega_{\Lambda^{c}}, s^{\prime} \in \Omega_{\Lambda^{\prime} c}$. Compare

$$
\langle F\rangle_{\Lambda}(s)-\langle F\rangle_{\Lambda^{\prime}}\left(s^{\prime}\right)=\sum_{s_{\Lambda}^{1}, s_{\Lambda^{\prime}}^{2}} \tilde{F} \nu\left(s_{\Lambda}^{1} \mid s\right) \nu\left(s_{\Lambda^{\prime}}^{2} \mid s^{\prime}\right)
$$

with $\Lambda, \Lambda^{\prime}$ such that $A \subset \Lambda \cap \Lambda^{\prime}$, and do the same expansion as above. The only terms that do not vanish are such that the connected component containing $\underline{A}$ intersects $\partial\left(\Lambda \cap \Lambda^{\prime}\right)$, because $\tilde{F}$ is antisymmetric under the $s^{1} \leftrightarrow s^{2}$ interchange. Hence, for each non-zero term, we may choose a path $P$ connecting $\underline{A}$ to $\partial\left(\Lambda \cap \Lambda^{\prime}\right)$. By the arguments given above, the sum over those paths is bounded by $\|F\| \exp \left(-m d\left(A, \partial\left(\Lambda \cap \Lambda^{\prime}\right)\right)\right)$, and (32) goes to zero as $\Lambda, \Lambda^{\prime} \uparrow \mathbf{Z}^{d}$.

In the next sections, we shall need a simple extension of Theorem 1 to a situation where the interactions depend (weakly) on $\Lambda$. Assume that, for each finite $\Lambda \subset \mathbf{Z}^{d}$, 
$\Phi_{\Lambda}=\Phi_{\Lambda}^{0}+\Phi_{\Lambda}^{1}$ where $\Phi_{\Lambda}^{0}, \Phi_{\Lambda}^{1}$ satisfy the hypotheses of Theorem 1 uniformly in $\Lambda$. Assume also that

$$
\left\|\Phi_{\Lambda X}-\Phi_{\Lambda^{\prime} X}\right\| \leq \epsilon e^{-\gamma d(X, \partial \Lambda)}
$$

for $X \subset \Lambda \subset \Lambda^{\prime}$, so that $\Phi_{X}=\lim _{\Lambda \uparrow \mathbf{Z}^{d}} \Phi_{\Lambda X}$ exists. Then we have

Theorem 2 Under the above assumptions, the conclusions of Theorem 1 hold; moreover, $\lim _{\Lambda \uparrow \mathbf{Z}^{d}} \nu_{\Lambda}=\mu$, where $\nu_{\Lambda}$ is defined by (2-4) with $\Phi_{X}$ replaced by $\Phi_{\Lambda X}$ and the sum in (2) is restricted to $X \subset \Lambda$.

Proof We study the limit $\Lambda \uparrow \mathbf{Z}^{d}$ of $\nu_{\Lambda}$. Let $\tilde{\Lambda} \subset \Lambda$ denote the largest cube containing the origin in $\mathbf{Z}^{d}$ such that $|\tilde{\Lambda}| \leq \frac{\gamma}{4} d(\Lambda)$ (so that $d(\tilde{\Lambda}) \rightarrow \infty$ if $\left.d(\Lambda) \rightarrow \infty\right)$. Write the Hamiltonian

$$
\mathcal{H}\left(s_{\Lambda}\right)=\sum_{X \subset \tilde{\Lambda}} \Phi_{X}+\Phi_{\tilde{\Lambda}}+\sum_{X \not \subset \tilde{\Lambda}} \Phi_{\Lambda X}
$$

where $\Phi_{\tilde{\Lambda}}=\sum_{X \subset \tilde{\Lambda}} \Phi_{\Lambda X}-\Phi_{X}$ can be regarded as an interaction coupling all the variables in $\tilde{\Lambda}$ and $\Phi_{X}=\lim _{\Lambda \uparrow \mathbf{Z}^{d}} \Phi_{\Lambda X}$. By (33), we have the trivial bound:

$$
\left\|\Phi_{\tilde{\Lambda}}\right\| \leq \sum_{X \subset \tilde{\Lambda}}\left\|\Phi_{\Lambda X}-\Phi_{X}\right\| \leq \epsilon 2^{|\tilde{\Lambda}|} e^{-\gamma d(\tilde{\Lambda}, \partial \Lambda)} \leq C \epsilon e^{-\frac{\gamma}{3} d(\tilde{\Lambda})} .
$$

So, $\Phi_{\tilde{\Lambda}}$ satisfies an estimate similar to the one of $\Phi^{1}$ (with a smaller $\gamma$ ). If we introduce this representation of $\mathcal{H}$ and expand $\Phi_{\tilde{\Lambda}}$, considering it as a part of $\Phi^{1}$, we see that the only non-zero term must contain a path connecting $A$ to $\partial \tilde{\Lambda}$. Since $d(\tilde{\Lambda}) \rightarrow \infty$ when $\Lambda \uparrow \mathbf{Z}^{d}$, the existence of the limit for $\nu_{\Lambda}$ follows. The rest is then as in the proof of Theorem 1.

Remark 1. We used in an essential way the fact that $\Phi$ is real when we used the positivity of $f_{X}, g_{X}, \mathcal{W}$. This can always be arranged for $\Phi$ real by adding a constant but not, of course, for complex $\Phi$. This "trick" of taking advantage of the positivity of the interactions was used e.g. in [2, 17, 20, 28]. The main interest of our method is that it yields uniqueness of the Gibbs state and exponential decay of correlations without using much combinatorics.

Remark 2. Here we first had $L$ determined by the $\Phi_{0}$ part of the interaction and then we chose $\epsilon$ small enough. However, in applications such as those of the next sections, the splitting of the interaction into $\Phi_{0}$ and $\Phi_{1}$ is somewhat arbitrary, and $L$ and $\epsilon$ are not independent. All we need is that $L^{d} \epsilon$ be small enough, see $(30,31)$.

Remark 3. The exponential decay (12) implies the decay of all the truncated correlation functions and, therefore, the fact the free energy and the correlation functions are $C^{\infty}$ (in the same sense as analyticity was defined above); see e.g. [40], Sect. II 12. Of course, 
the bounds obtained in this way on the truncated correlation functions contain factorials that prevent us from drawing any conclusions about their analyticity.

Remark 4. Since we used $\left\|\Phi^{1}\right\|_{2} \leq \epsilon$ only in the bounds $(19,30)$, we can easily extend our results in several directions: First, one recovers uniqueness of the Gibbs state if we assume only $\left\|\Phi^{1}\right\|_{1} \leq \epsilon$, and $\Phi^{0}$ as before. Instead of (19), we have

$$
\sum_{0 \in X}|X| \| f_{X}|| \leq \mathcal{O}(\epsilon)
$$

and (30) holds without the factor $\exp \left(\frac{\gamma}{2} d(X)\right)$. Taking $\Phi^{0}=0$, this yields a simple proof of Dobrushin's uniqueness theorem. Using $(30,31)$ (without $\left.\exp \left(\frac{\gamma}{2} d(X)\right)\right)$ we can also bound

$$
\sum_{i \in \mathbf{Z}^{d}}\left|\left\langle F \tau_{i} G\right\rangle-\langle F\rangle\langle G\rangle\right|<\infty
$$

(just sum over $Z_{n}=\tau_{i} \underline{B}$ ). Therefore, one recovers the result of Gross [24] on the $C^{2}$ property of the free energy.

We may also consider potentials decreasing with a power law: introduce the norm

$$
\|\Phi\|_{4}=\sum_{0 \in X}|X| e^{\gamma \ln d(X)}\left\|\Phi_{X}\right\|
$$

Then, if $\left\|\Phi^{1}\right\|_{4}$ is small, we get an upper bound $d(A, B)^{-\gamma}$ in (12). Of course, here we need the factor $|X|$ in (36) to control the sum (30).

Finally, let us remark that we do not use the translation invariance of $\Phi$ except when we speak of the free energy, of analyticity or of differentiability. Hence, uniqueness of the Gibbs state and the decay of correlation (12) hold for arbitrary interactions on arbitrary lattices with

$$
\sup _{i} \sum_{i \in X}|X|\left\|\Phi_{X}^{1}\right\| \text { or } \sup _{i} \sum_{i \in X} e^{\gamma d(X)}\left\|\Phi_{X}^{1}\right\|
$$

small enough.

\section{Coupled map lattices.}

We consider the following class of dynamical systems. The phase space $\mathcal{M}=\left(S^{1}\right)^{\mathbf{Z}^{d}}$ i.e. $\mathcal{M}$ is the set of maps $\mathbf{z}=\left(z_{j}\right)_{j \in \mathbf{Z}^{d}}$ from $\mathbf{Z}^{d}$ to the circle. $\mathcal{M}$ inherits its topology and Borel $\sigma$-algebra $\mathcal{B}$ from $S^{1}$ : it is a compact metric space and we let $m$ denote the product of Lebesgue measures on the $S^{1}$-factors. $\mathcal{M}$ carries a natural $\mathbf{Z}^{d}$-action, denoted by $\tau_{i}, i \in \mathbf{Z}^{d}$.

To describe the dynamics, we first fix a map $F: S^{1} \rightarrow S^{1}$. We take $F$ to be an expanding, orientation preserving $C^{1+\delta}$ map with $\delta>0$. We describe $F$ in terms of its lift to $\mathbf{R}$, denoted by $f$ and chosen, say, with $f(0) \in[0,1[$. We assume that

$$
f^{\prime}(x)>\lambda^{-1}
$$


where $\lambda<1$. Note that there exists an integer $k>1$ such that

$$
f(x+1)=f(x)+k \quad \forall x \in \mathbf{R}
$$

We let $\mathcal{F}: \mathcal{M} \rightarrow \mathcal{M}$ denote the Cartesian product $\mathcal{F}=\mathrm{X}_{i \in \mathbf{Z}^{d}} F_{i}$ where $F_{i}$ is a copy of $F$. $\mathcal{F}$ is called the uncoupled map.

The second ingredient in the dynamics is given by the coupling map $A: \mathcal{M} \rightarrow \mathcal{M}$. This is taken to be a small perturbation of the identity in the following sense. Let $A_{i}$ be the projection of $A$ on the $\mathrm{i}^{\text {th }}$ factor. We assume that $A_{i}$ is $C^{1}$ in each $z_{j}$ and satisfies (we use the parametization $z_{j}=e^{2 \pi i x_{j}}$ on $S^{1}, x_{j} \in\left[0,1\left[\right.\right.$ and we let $a_{j}$ denote the lift of $\left.A_{j}: A_{j}=e^{2 \pi i a_{j}}\right)$ :

$$
\left\|\frac{\partial a_{i}}{\partial x_{j}}-\delta_{i j}\right\|_{\infty} \leq \epsilon e^{-\beta|i-j|}
$$

together with a Hölder condition

$$
\left|\frac{\partial a_{i}}{\partial x_{j}}(\mathbf{x})-\frac{\partial a_{i}}{\partial x_{j}}(\mathbf{y})\right| \leq \epsilon \sum_{k} e^{-\beta(|i-j|+|i-k|)}\left|x_{k}-y_{k}\right|^{\delta}
$$

for some $\epsilon, \beta, \delta>0$, with $\mathbf{x}=\left(x_{i}\right)_{i \in \mathbf{Z}^{d}}$.

Furthermore, we suppose that $A$ is $\mathbf{Z}^{d}$-invariant:

$$
A=\tau_{i} \circ A \circ \tau_{i}^{-1} \forall i \in \mathbf{Z}^{d},
$$

although this is not essential, see Remark 2 in [3] and Remark 4 at the end of Section 2.

Example. An example often considered (see e.g. [3]) is the coupling map

$$
a_{j}(\mathbf{x})=x_{j}+2 \pi i \epsilon \sum_{k} g_{|j-k|}\left(x_{j}, x_{k}\right)
$$

where $g$ is a periodic $C^{1+\delta}$ function in both variables, with exponential falloff in $|j-k|$ as in (4). More general examples of such $A^{\prime} s$ can be found in [3] (where, however, we restricted ourselves to analytic maps).

The coupled map $T: \mathcal{M} \rightarrow \mathcal{M}$ is now defined by

$$
T=A \circ \mathcal{F} .
$$

We are looking for "natural" $T$-invariant measures on $\mathcal{M}$. For this, write, for $\Lambda \subset \mathbf{Z}^{d}$, $\mathcal{M}_{\Lambda}=\left(S^{1}\right)^{\Lambda}$, and let $m_{\Lambda}$ be the product of Lebesgue measures. Let $\mathcal{B}_{\Lambda} \subset \mathcal{B}$ be the $\sigma$-algebra of subsets of $\mathcal{M}$ generated by the Borel sets of $\mathcal{M}_{\Lambda}$ (identified in the obvious way with subsets of $\mathcal{M}$ ).

Definition 1 A Borel probability measure $\mu$ on $\mathcal{B}$ is a SRB measure if

(a) $\mu$ is T-invariant 
(b) The restriction $\mu_{\Lambda}$ of $\mu$ to $\mathcal{B}_{\Lambda}$ is absolutely continuous with respect to $m_{\Lambda}$ for all $\Lambda \subset \mathbf{Z}^{d}$ finite.

Remark This is a natural extension of the notion of SRB measure to infinite dimensions, since each $S^{1}$ factor can be regarded as an unstable direction.

It is convenient to introduce the space of Hölder continuous functions $\mathcal{C}^{\delta}\left(\mathcal{M}_{\Lambda}\right)$ for $\Lambda \subset \mathbf{Z}^{d},|\Lambda|<\infty$ equipped with the norm

$$
\|G\|_{\delta}=\|G\|_{\infty}+\sup _{\mathbf{x}, \mathbf{y}} \frac{|G(\mathbf{x})-G(\mathbf{y})|}{\sum_{i \in \Lambda}\left|x_{i}-y_{i}\right|^{\delta}}
$$

We also write $\mathcal{C}\left(\mathcal{M}_{\Lambda}\right)$ to denote the continuous functions on $\mathcal{M}_{\Lambda}$ with the sup norm.

Definition $2 A$ Borel probability measure on $\mathcal{B}$ is regular if its restriction $\mu_{\Lambda}$ to $\mathcal{B}_{\Lambda}$ is absolutely continuous with respect to $m_{\Lambda}$ for all $\Lambda \subset \mathbf{Z}^{d}$ finite and if $\log h_{\Lambda} \in \mathcal{C}^{\delta}\left(\mathcal{M}_{\Lambda}\right)$, where $h_{\Lambda}=\frac{d \mu_{\Lambda}}{d m_{\Lambda}}$ :

$$
\left|\log \frac{h_{\Lambda}(\mathbf{x})}{h_{\Lambda}(\mathbf{y})}\right| \leq C \sum_{i \in \Lambda}\left|x_{i}-y_{i}\right|^{\delta}
$$

Remark A similar condition was introduced by Volevich [44]. If $\mu$ is a Gibbs state on $\mathcal{M}$ for some translation invariant interaction such that $\sum_{0 \in X}\left\|\Phi_{X}\right\|<\infty$ and

$$
\left|\Phi_{X}(\mathbf{x})-\Phi_{X}(\mathbf{y})\right| \leq C(X) \sum_{i \in X}\left|x_{i}-y_{i}\right|^{\delta}
$$

with

$$
\sum_{0 \in X} C(X)<\infty
$$

then $\mu$ is regular. This follows easily from the DLR equations:

$$
h_{\Lambda}(\mathbf{x})=\int \nu(\mathbf{x} \mid \mathbf{z}) d \mu(\mathbf{z})
$$

where $\nu(\mathbf{x} \mid \mathbf{z})$ is defined as in (2.3). To get (6), using (9), by Jensen's inequality it is enough to bound

$$
\begin{aligned}
& \sup _{\mathbf{z}}\left|\log \frac{\nu(\mathbf{x} \mid \mathbf{z})}{\nu(\mathbf{y} \mid \mathbf{z})}\right|=\sup _{\mathbf{z}}|\mathcal{H}(\mathbf{x} \mid \mathbf{z})-\mathcal{H}(\mathbf{y} \mid \mathbf{z})| \\
& \leq \sup _{\mathbf{z}} \sum_{X \cap \Lambda \neq \phi}\left|\Phi_{X}(\mathbf{x} \vee \mathbf{z})-\Phi_{X}(\mathbf{y} \vee \mathbf{z})\right|
\end{aligned}
$$

which, using $(7,8)$, is bounded by the RHS of (6).

We will prove 
Theorem 3 Let $F$ and $A$ satisfy the assumptions given above. Then there exists $\epsilon_{0}>0$ such that, for $\epsilon<\epsilon_{0}$, T has an SRB measure $\mu$. Furthermore, $\mu$ is invariant and exponentially mixing under the space-time translations generated by $\tau_{i}$ and $T$ : there exists $m>0, C<\infty$, such that, $\forall B, D \subset \mathbf{Z}^{d},|B|,|D|<\infty$ and $\forall G \in L^{\infty}\left(\mathcal{M}_{B}\right), \forall H \in$ $\mathcal{C}^{\delta}\left(\mathcal{M}_{D}\right)$

$$
\left|\int G \circ T^{n} H d \mu-\int G d \mu \int H d \mu\right| \leq C\|G\|_{\infty}\|H\|_{\delta} \min (|B|,|D|) e^{-m(n+d(B, D))},
$$

where $d(B, D)$ is the distance between $B$ and $D$.

Finally, for any regular measure $\nu, T^{* N} \nu \rightarrow \mu$ weakly, as $N \rightarrow \infty$, $T^{*}$ being the transpose of $T$.

Remark. We shall construct an expansion for the Perron-Frobenius operator of $T$, which will be quite similar to the expansion of the previous section, for $\Phi$ of the form (2.9), with $\Phi^{0}$ one-dimensional and $\left\|\Phi^{1}\right\|_{2}$ small. Bunimovich and Sinai [5] have reduced the problem (for a one dimensional lattice of maps, and a slightly different model) to a spin system with exponentially decaying interactions of the same form. We shall rederive this estimate below (see Proposition 2). However, they did not discuss in detail the resulting statistical mechanical system (the reference to 12 is slightly misleading, since the latter authors assume $\left\|\Phi^{1}\right\|_{3}$ small, which probably does not hold in general here). The work of Bunimovich and Sinai was extended by Volevich [44, 45, 46], Pesin

and Sinai [36], and by Jiang [27]. Our results are quite close to those of [46], where lattices of dimension $d>1$ are considered. In that paper, the statistical mechanics is based on an extension to infinite range interactions of results of Dobrushin and Pecherski |14.

An extension of [5] is also claimed in [25, 8]. However, there the treatment of the statistical mechanics part of the problem is wrong. The authors essentially claim that uniqueness of the Gibbs state holds for any $\Phi$, with $\|\Phi\|_{2}$ finite. Although this is true in one dimension, it certainly does not hold in higher dimensions. The Ising model, or any finite range model of this type that undergoes a phase transition, provide counterexamples. Moreover, the "proof" would also yield analyticity, which does not hold even under the correct assumption that $\Phi$ is of the form (2.9), with $\Phi^{0}$ one dimensional and $\left\|\Phi^{1}\right\|_{2}$ small, because of the counterexamples in 13.

Finally, in [3], we constructed a convergent expansion for the Perron-Frobenius operator, but we needed stronger assumptions on the coupled map lattice (analyticity instead of smoothness). These assumptions, in the present context, would imply that $\left\|\Phi^{1}\right\|_{3}$ is small and that we could use the usual cluster expansion.

\section{SRB measure from a Gibbs measure}

The SRB measure $\mu$ is constructed using the Perron-Frobenius operator for $T$. We first introduce a finite volume cutoff version of $T, T_{\Lambda}: \mathcal{M}_{\Lambda} \rightarrow \mathcal{M}_{\Lambda}, \Lambda \subset \mathbf{Z}^{d},|\Lambda|<\infty$. For definiteness, we let $\Lambda$ be a cube centered at 0 . Set $T_{\Lambda}=A_{\Lambda} \circ \mathcal{F}_{\Lambda}$ with $\mathcal{F}_{\Lambda}=\times_{i \in \Lambda} F_{i}$ and

$$
A_{\Lambda}=R_{\Lambda} \circ A \circ \mathcal{C}_{\Lambda}
$$


where $\mathcal{C}_{\Lambda}: \mathcal{M}_{\Lambda} \rightarrow \mathcal{M}$ extends $\mathbf{z} \in \mathcal{M}_{\Lambda}$ to $\mathcal{M}$ by

$$
\mathcal{C}_{\Lambda}(\mathbf{z})_{i}=\left\{\begin{array}{cc}
z_{i} & i \in \Lambda \\
1 & i \notin \Lambda
\end{array}\right.
$$

(here $z_{i}, 1 \in S^{1}$, and the choice of 1 is arbitrary), and $R_{\Lambda}: \mathcal{M} \rightarrow \mathcal{M}_{\Lambda}$ is the restriction. It is more convenient to work in the covering space $\mathbf{R}^{\Lambda}$ of $\mathcal{M}_{\Lambda}$ and to introduce the corresponding lifts $a_{\Lambda}, f_{\Lambda}, t_{\Lambda}$ as maps from $\mathbf{R}^{\Lambda}$ to itself. Our assumptions for $A$ then imply the following properties for $a_{\Lambda}$.

Lemma 1 The maps $a_{\Lambda}$ are $C^{1+\delta}$ diffeomeorphisms with the following properties: there exist $\beta>0, C<\infty$, independent of $\Lambda$, such that $\forall \mathbf{x} \in \mathbf{R}^{\Lambda}$

a) $a_{\Lambda}(\mathbf{x}+\mathbf{n})=a_{\Lambda}(\mathbf{x})+\mathbf{n} \forall \mathbf{n} \in \mathbf{Z}^{\Lambda}$

b) The derivative of $a_{\Lambda}$ can be written as $D a_{\Lambda}(\mathbf{x})=I+\epsilon_{\Lambda}(\mathbf{x})$, where $I$ is the unit matrix, and $\epsilon_{\Lambda}$ is periodic function satisfying

$$
\begin{gathered}
\left|\epsilon_{\Lambda}(\mathbf{x})_{i j}\right| \leq C \epsilon e^{-\beta|i-j|} \\
\left|\epsilon_{\Lambda}(\mathbf{x})_{i j}-\epsilon_{\Lambda}(\mathbf{y})_{i j}\right| \leq C \epsilon \sum_{k \in \Lambda} e^{-\beta(|i-j|+|i-k|)}\left|x_{k}-y_{k}\right|^{\delta}
\end{gathered}
$$

c) $\epsilon_{\Lambda}(\mathbf{x})_{i j}=\epsilon_{\Lambda^{\prime}}\left(\mathbf{x} \vee \mathbf{1}_{\Lambda^{\prime} \backslash \Lambda}\right)_{i j}$ for $i, j \in \Lambda \subset \Lambda^{\prime}$.

d) Let $\Lambda \subset \Lambda^{\prime}, R_{\Lambda} \mathbf{y}=\mathbf{x}, \mathbf{y} \in \mathbf{R}^{\Lambda^{\prime}}$ and $i, j \in \Lambda$. Then

$$
\left|\epsilon_{\Lambda}(\mathbf{x})_{i j}-\epsilon_{\Lambda^{\prime}}(\mathbf{y})_{i j}\right| \leq C \epsilon e^{-\beta|i-j|} e^{-\beta d(i, \partial \Lambda)}
$$

where d denotes the distance.

Furthermore $a_{\Lambda}^{-1}$ satisfies a)-d) also, with possibly different $\beta, C$.

Proof (2) and (3) are just a restatement of (3.3), (3.4) (we can take $C=1$ here). By definition of a lift, $a_{\Lambda}(\mathbf{x}+\mathbf{n})=a_{\Lambda}(\mathbf{x})+\mathbf{m}(\mathbf{n})$ for some $\mathbf{m}(\mathbf{n}) \in \mathbf{Z}^{\Lambda}$. Write $a_{\Lambda}=i d+b$ where $i d$ is the identity. Order points of $\Lambda$ in an arbitrary way such that $\Lambda=\left\{i_{\ell} \mid \ell=\right.$ $1, \cdots,|\Lambda|\}$. Then, given $\mathbf{x}, \mathbf{y} \in \mathbf{R}^{\Lambda}$, define $\mathbf{w}^{(n)}$ by letting let $w_{i_{\ell}}^{(n)}$ equal $x_{i_{\ell}}$ for $\ell \geq n$ and $y_{i_{\ell}}$ for $\ell<n$. Then,

$$
b_{i}(\mathbf{x})-b_{i}(\mathbf{y})=\sum_{n=1}^{|\Lambda|} b_{i}\left(\mathbf{w}^{(n)}\right)-b_{i}\left(\mathbf{w}^{(n+1)}\right)
$$

and, from (2),

$$
\begin{aligned}
\left|b_{i}\left(\mathbf{w}^{(n)}\right)-b_{i}\left(\mathbf{w}^{(n+1)}\right)\right| & \leq \int_{0}^{1} d t\left|\epsilon_{i i_{n}}\left((1-t) \mathbf{w}^{(n)}+t \mathbf{w}^{(n+1)}\right)\right|\left|x_{i_{n}}-y_{i_{n}}\right| \\
& \leq C \epsilon e^{-\beta\left|i-i_{n}\right|}\left|x_{i_{n}}-y_{i_{n}}\right|
\end{aligned}
$$


Hence,

$$
\left|b_{i}(\mathbf{x})-b_{i}(\mathbf{y})\right| \leq C \epsilon \sum_{j \in \Lambda} e^{-\beta|i-j|}\left|x_{j}-y_{j}\right|
$$

Let $\left|n_{j}\right| \leq 1$. Then, for $\epsilon$ small, $\mathbf{m}(\mathbf{n})=\mathbf{n}$ and thus by iteration a) holds for all $\mathbf{n}$. Point c) follows from the definition (1) and d) follows from c) and (3).

It is now straightforward to show that $a_{\Lambda}^{-1}$ exists and satisfies a)-d) too, (possibly with different $\beta, C)$ : Writing $D a_{\Lambda}^{-1}=1+\tilde{\epsilon}_{\Lambda}=\left(1+\epsilon_{\Lambda}\right)^{-1}$ and using (2) with $C=1$, we get:

$$
\left|\tilde{\epsilon}_{\Lambda}(\mathbf{x})_{i j}\right| \leq \sum_{n=1}^{\infty}\left|\epsilon_{\Lambda}^{n}(\mathbf{x})_{i j}\right| \leq \sum_{n=1}^{\infty} \epsilon^{n} \sum_{i_{2}, \cdots, i_{n-1}} \prod_{\ell=1}^{n-1} e^{-\beta\left|i_{\ell}-i_{\ell+1}\right|}
$$

where $i_{1}=i, i_{n}=j$. This is readily bounded by $\epsilon e^{-\tilde{\beta}|i-j|}$ for any $\tilde{\beta}<\beta$ and $C=C(\tilde{\beta})<$ $\infty$. This proves (2) for $a_{\Lambda}^{-1}$. To prove (3) for $a_{\Lambda}^{-1}$, we use (3) for $\epsilon_{\Lambda}$ and

$$
\left|\epsilon_{\Lambda}^{n}(\mathbf{x})_{i j}-\epsilon_{\Lambda}^{n}(\mathbf{y})_{i j}\right| \leq \epsilon^{n} \sum_{i_{2}, \cdots, i_{n-1}} \sum_{\ell=1}^{n} \sum_{k} e^{-\beta\left|i_{\ell}-k\right|} \prod_{\ell=1}^{n-1} e^{-\beta\left|i_{\ell}-i_{\ell+1}\right|}\left|x_{k}-y_{k}\right|^{\delta}
$$

with $i_{1}=i, i_{n}=j$, which is bounded by

$$
\leq(C \epsilon)^{n} \sum_{k} e^{-\tilde{\beta}(|i-j|+|i-k|)}\left|x_{k}-y_{k}\right|^{\delta}
$$

with $\tilde{\beta}<\frac{\beta}{2}$ and $C$ as above. The proof of (4) is similar.

The Perron-Frobenius operator $P_{\Lambda}$ for $T_{\Lambda}$ is defined as usual by

$$
\int G \circ T_{\Lambda} H d m_{\Lambda}=\int G P_{\Lambda} H d m_{\Lambda}
$$

for $G \in L^{\infty}\left(\mathcal{M}_{\Lambda}\right), H \in L^{1}\left(\mathcal{M}_{\Lambda}\right)$. Let us work in the covering space $\mathbf{R}^{\Lambda}$ and replace $G, H$ by periodic functions denoted $g, h: g(\mathbf{x}+\mathbf{n})=g(\mathbf{x}), \forall \mathbf{n} \in \mathbf{Z}^{\Lambda}$. Insert $t_{\Lambda}=a_{\Lambda} \circ f_{\Lambda}$ in (8) to get (with $C_{\Lambda}=[0,1]^{\Lambda}$ )

$$
\int_{C_{\Lambda}} g \circ t_{\Lambda} h d \mathbf{x}=\int_{C_{\Lambda}} g\left(a_{\Lambda}(\mathbf{x})\right) P_{\Lambda}^{\circ} h(\mathbf{x}) d \mathbf{x}
$$

where $P_{\Lambda}^{\circ}$ is the Perron-Frobenius operator for $f_{\Lambda}$ i.e.

$$
P_{\Lambda}^{\circ} h(\mathbf{x})=\sum_{\mathbf{s}} \frac{h\left(f_{\Lambda}^{-1}(\mathbf{x}+\mathbf{s})\right)}{\prod_{i \in \Lambda} f^{\prime}\left(f^{-1}\left(x_{i}+s_{i}\right)\right)}
$$

where $\mathbf{s} \in\{0, \cdots, k-1\}^{\Lambda}$ (and $k$ was introduced in (3.2)). Note that $P_{\Lambda}^{\circ}$ maps periodic functions into periodic functions because the sum is periodic even if the summands are not: indeed, (3.2) implies that $f^{-1}(x+1+k-1)=f^{-1}(x+k)=f^{-1}(x)+1$ (so that, if we add 1 to $x_{i}$, it amounts to a cyclic permutation of $s_{i}$ ) and that $f^{\prime}$ is periodic. 
Since, for a periodic function $k$

$$
\int_{a_{\Lambda}\left(C_{\Lambda}\right)} k(\mathbf{x}) d \mathbf{x}=\int_{C_{\Lambda}} k(\mathbf{x}) d \mathbf{x}
$$

(both $C_{\Lambda}$ and $a_{\Lambda}\left(C_{\Lambda}\right)$ are, up to their boundaries, fundamental domains for the action of $\mathbf{Z}^{\Lambda}$ on $\mathbf{R}^{\Lambda}$ ), we obtain the formula

$$
P_{\Lambda} h(\mathbf{x})=\operatorname{det} D a_{\Lambda}^{-1}(\mathbf{x}) \sum_{\mathbf{s}} \frac{h\left(\psi_{\Lambda \mathbf{s}}(\mathbf{x})\right)}{\prod_{i \in \Lambda} f^{\prime}\left(\psi_{\Lambda \mathbf{s}}(\mathbf{x})_{i}\right)}
$$

where we defined

$$
\psi_{\Lambda \mathbf{s}}(\mathbf{x})_{i}=f^{-1}\left(a_{\Lambda}^{-1}(\mathbf{x})_{i}+s_{i}\right) .
$$

and (with a slight abuse of notations) we write $P_{\Lambda}$ for the operator acting on periodic functions induced by the Perron-Frobenius operator defined by (8). Note that, by Lemma 4.1.a, $a_{\Lambda}^{-1}(\mathbf{x}+\mathbf{n})=a_{\Lambda}^{-1}(\mathbf{x})+\mathbf{n}$ and, so, $P_{\Lambda}$ maps also periodic functions into periodic functions.

The invariant measure $\mu$ is constructed as a weak limit of the measures $T_{\Lambda}^{* N} m_{\Lambda}$, with $T_{\Lambda}^{*}$ being the transpose of $T_{\Lambda}$, as $N \rightarrow \infty$ and $\Lambda \uparrow \mathbf{Z}^{d}$. These in turn are given by the Perron-Frobenius operator

$$
T_{\Lambda}^{* N} m_{\Lambda}=\left(P_{\Lambda}^{N} 1\right) m_{\Lambda}
$$

by putting $H=1$ in (8). $P_{\Lambda}^{N} 1$ has a direct statistical mechanical interpretation which we now derive.

First, iterating (10), we get

$$
\left(P_{\Lambda}^{N} 1\right)(\mathbf{x})=\sum_{\mathbf{s}_{1}, \cdots \mathbf{s}_{N}} \prod_{t=1}^{N}\left[\operatorname{det} D a_{\Lambda}^{-1}\left(\psi_{\mathbf{s}_{t-1}} \circ \cdots \circ \psi_{\mathbf{s}_{1}}(\mathbf{x})\right) \prod_{i \in \Lambda}\left(f^{\prime}\left(\psi_{\mathbf{s}_{t}} \circ \cdots \circ \psi_{\mathbf{s}_{1}}(\mathbf{x})_{i}\right)\right)^{-1}\right]
$$

where, for $t=1$, the argument of $D a_{\Lambda}^{-1}$ is $\mathbf{x}$, and we write $\psi_{\mathbf{s}}$ for $\psi_{\Lambda \mathbf{s}}$. From now on, we shall consider $\mathbf{x} \in C_{\Lambda}$.

Next, we introduce a convenient notation: $\mathbf{x} \in C_{\Lambda}$ and $\mathbf{s}_{1}, \cdots, \mathbf{s}_{N}$ in (12) collectively define a configuration on a "space-time" lattice $\{0, \cdots, N\} \times \Lambda$. Thus, let $\mathbf{Z}_{+}^{d+1}=$ $\mathbf{Z}_{+} \times \mathbf{Z}^{d}, \mathbf{Z}_{+}$being the non-negative integers. To any subset $X \subset \mathbf{Z}_{+}^{d+1}$ associate the configuration space $\Omega_{X}=\times_{\alpha \in X} \Omega_{\alpha}$ where, for $\alpha=(t, i), \Omega_{\alpha}$ equals $[0,1]$ if $t=0$, and equals $\{0, \cdots, k-1\}$ if $t>0$. We could use the existence of a Markov partition for $T_{\Lambda}$ to write $\mathbf{x}$ as a symbol sequence, as is usually done, e.g. in [5], but we shall not need this representation.

Let $\Lambda_{N}=\{0, \cdots, N\} \times \Lambda$ and $s=\left(\mathbf{x}, \mathbf{s}_{1}, \cdots, \mathbf{s}_{N}\right) \in \Omega_{\Lambda_{N}}$. We shall write $\mathbf{x}, \mathbf{s}$, as before, for elements of $\Omega_{\Lambda}, \Lambda \subset \mathbf{Z}^{d}$, and use $s$ to denote elements of $\Omega_{\Lambda}, \Lambda \subset \mathbf{Z}_{+}^{d+1}$. Then (12) reads

$$
\left(P_{\Lambda}^{N} 1\right)(\mathbf{x})=\sum_{\mathbf{s}_{1} \cdots \mathbf{s}_{N}} e^{-\mathcal{H}_{\Lambda_{N}}(s)}
$$

with $e^{-\mathcal{H}_{\Lambda_{N}}}$ being the summand in (12). 
The final step is to write $\mathcal{H}_{\Lambda_{N}}$ in terms of potentials. First, write $D a_{\Lambda}^{-1}=1+\tilde{\epsilon}_{\Lambda}$, where, by Lemma 1, $\tilde{\epsilon}_{\Lambda}$ satisfies (2)-(4). Then expand

$$
\operatorname{det} D a_{\Lambda}^{-1}=\operatorname{det}\left(1+\tilde{\epsilon}_{\Lambda}\right)=\exp \left[-\sum_{i \in \Lambda} v_{\Lambda i}\right]
$$

where

$$
v_{\Lambda i}(\mathbf{x})=\sum_{n=1}^{\infty} \frac{(-1)^{n}}{n}\left(\tilde{\epsilon}_{\Lambda}(\mathbf{x})^{n}\right)_{i i}
$$

Lemma 2 There exist $\epsilon_{1}>0, C<\infty$ such that, for $\epsilon<\epsilon_{1}$,

$$
\left\|v_{\Lambda i}\right\|_{\infty} \leq C \epsilon
$$

and

$$
\left|v_{\Lambda i}(\mathbf{x})-v_{\Lambda i}(\mathbf{y})\right| \leq C \epsilon \sum_{k \in \Lambda} e^{-\frac{\beta}{2}|i-k|}\left|x_{k}-y_{k}\right|^{\delta}
$$

and, for $\Lambda \subset \Lambda^{\prime}, \mathbf{x}=R_{\Lambda} \mathbf{y}, i \in \Lambda$

$$
\left|v_{\Lambda i}(\mathbf{x})-v_{\Lambda^{\prime} i}(\mathbf{y})\right| \leq C \epsilon e^{-\frac{\beta}{2} d(i, \partial \Lambda)}
$$

uniformly in $\Lambda, \Lambda^{\prime}$.

Proof Straightforward, using (2)-(4), and the bound (7), for $\tilde{\epsilon}_{\Lambda}$.

Given $\alpha=(t, i) \in \Lambda_{N}$ we set

$$
V_{\Lambda \alpha}(s)=v_{\Lambda i}\left(\psi_{\mathbf{s}_{t-1}} \circ \cdots \circ \psi_{\mathbf{s}_{1}}(\mathbf{x})\right)+\log f^{\prime}\left(\left(\psi_{\mathbf{s}_{t}} \circ \cdots \circ \psi_{\mathbf{s}_{1}}(\mathbf{x})\right)_{i}\right)
$$

and hence

$$
\mathcal{H}_{\Lambda_{N}}(s)=\sum_{\alpha \in \Lambda_{N}} V_{\Lambda \alpha}(s)
$$

where $V_{\Lambda \alpha}$ depends on $\left.s\right|_{\Lambda_{N}}$. The representation (16) still needs to be localized in order to express $\mathcal{H}$ in terms of potentials. We use the notation of Section 2: given $s \in \Omega_{X}$, $s^{\prime} \in \Omega_{Y}$ for $X \cap Y=\emptyset$ we denote by $s \vee s^{\prime}$ the corrresponding configuration in $X \cup Y$, and, if $Z \subset X$, we let $s_{Z}=\left.s\right|_{Z}$. Also, let $0_{X} \in \Omega_{X}$ be $\left(0_{X}\right)_{\alpha}=0, \forall \alpha \in X$ (note that 0 belongs to $\Omega_{\alpha}$ for any $\alpha$ ).

Now, given $t$, choose an arbitrary ordering of the points of $\{0, \ldots, t\} \times \mathbf{Z}^{d}=\left\{\alpha_{\ell} \mid \ell \in\right.$ $\mathbf{N}\}$ in such a way that $\left|\alpha_{\ell}\right|$ is a non-decreasing function of $\ell$ where, for $\alpha=\left(t^{\prime}, j\right)$

$$
|\alpha|=\frac{1}{M}\left|t^{\prime}\right|+\sum_{k=1}^{d}\left|j_{k}\right| .
$$

$M$ will be taken sufficiently large below (see (25)). 
Consider $V_{\Lambda \alpha}$ in (16). Let $Y_{\alpha}^{m}=\left\{\alpha-\alpha_{\ell} \mid 0 \leq \ell \leq m\right\}$, and set, for $s \in \Omega_{Y_{\alpha}^{m}}$

$$
\Phi_{Y_{\alpha}^{m}}^{\Lambda}(s)=V_{\Lambda \alpha}\left(s_{Y_{\alpha}^{m}} \vee 0_{\left(Y_{\alpha}^{m}\right)^{c}}\right)-V_{\Lambda \alpha}\left(s_{Y_{\alpha}^{m-1}} \vee 0_{\left(Y_{\alpha}^{m-1}\right)^{c}}\right)
$$

Note that the two configurations on the RHS of (18) differ only at site $\alpha-\alpha_{m}$. We have then the identity

$$
V_{\Lambda \alpha}(s)=\sum_{m \geq 0} \Phi_{Y_{\alpha}^{m}}^{\Lambda}(s)+V_{\Lambda \alpha}(0)
$$

and we define

$$
\Phi_{\Lambda X}(s)=\Phi_{Y_{\alpha}^{m}}^{\Lambda}(s)
$$

provided there exists $m, \alpha$ such that $X=Y_{\alpha}^{m}$, otherwise we set $\Phi_{\Lambda X}(s)=0$ (note that there is at most one such pair $(n, \alpha))$. $\Phi_{\Lambda X}$ depends only on $s_{X}$. Due to (16), (19), we have

$$
\mathcal{H}_{\Lambda_{N}}(s)=\sum_{X \subset \Lambda_{N}} \Phi_{\Lambda X}(s)+\text { constant }
$$

where constant $=\sum_{\alpha \in \Lambda_{N}} V_{\Lambda \alpha}(0)$. Note that (17) was chosen so that $Y_{\alpha}^{m}$ with $\left|\alpha_{m}\right|<1$ is a one-dimensional interval of length at most $M$ in the time direction.

Now write

$$
\Phi_{\Lambda}=\Phi_{\Lambda}^{0}+\Phi_{\Lambda}^{1}
$$

where $\Phi^{0}$ collects the $X^{\prime} s$ with $\left|\alpha_{m}\right|<1$ in (20) and $\Phi^{1}$ all the others. We have the following basic bound:

Proposition 1 There exist $\epsilon_{2}>0, C<\infty$, such that, if $\epsilon<\epsilon_{2}$, then $\Phi_{Y_{\alpha}^{m}}$ given by (18) is bounded by

$$
\left|\Phi_{Y_{\alpha}^{m}}^{\Lambda}(s)\right| \leq C \epsilon^{\prime \delta_{0 i m}} \lambda^{\prime t_{m}} e^{-\beta^{\prime}\left|i_{m}\right|}
$$

where $\alpha_{m}=\left(t_{m}, i_{m}\right) \lambda^{\prime}=\lambda^{\delta / 2}, \epsilon^{\prime}=\epsilon^{\delta}, \beta^{\prime}=\frac{\beta \delta}{3}$ where $\delta$ is as in (3.4). Moreover, for any $\Lambda^{\prime} \supset \Lambda$,

$$
\left|\Phi_{Y_{\alpha}^{m}}^{\Lambda}(s)-\Phi_{Y_{\alpha}^{m}}^{\Lambda^{\prime}}(s)\right| \leq C \epsilon^{\prime} e^{-\beta^{\prime} d\left(Y_{\alpha}^{m}, \partial \Lambda\right)}
$$

Choose now $M$ in (17) to be the smallest integer such that

$$
\lambda^{\prime M}<\epsilon^{\delta} .
$$

Then, we have 
Proposition 2 There exist $\gamma>0, \epsilon_{0}>0, C<\infty$ such that, if $\epsilon<\epsilon_{0}$, $\Phi^{1}$ satisfies the bound

$$
\sum_{\alpha \in X} e^{\gamma d(X)}\left\|\Phi_{\Lambda X}^{1}\right\| \leq C \epsilon^{\delta}
$$

uniformly in $\alpha$ and $\Lambda$. Moreover,

$$
\left|\Phi_{\Lambda X}(s)-\Phi_{\Lambda^{\prime} X}(s)\right| \leq C \epsilon^{\delta} e^{-\gamma d(X, \partial \Lambda)} .
$$

with $X \subset \Lambda \subset \Lambda^{\prime}$.

Since $\Phi^{0}$ has no coupling in spatial directions and has range $M$ in the time direction we get the following decomposition of its partition function $Z^{0}$. We let for $i \in \mathbf{Z}^{d}$ $J_{i}=\left(\mathbf{Z}_{+} \times i\right) \cap V$ so $V=\cup_{i} J_{i}$. Then

$$
Z^{0}(V \mid s)=\prod_{i} Z^{0}\left(J_{i} \mid s\right)
$$

Proposition 3 There exist $\gamma>0, C<\infty$ independent of $M$ such that, if $J$ is an interval of the form $[(i, t),(i, t+\ell M)]$ then

$$
Z^{0}(J \mid s)=\lambda^{\ell} W\left(s_{-}\right) W\left(s_{+}\right)\left(1+g_{J}\left(s_{-}, s_{+}\right)\right)
$$

where $s_{+}=s_{((i, t+\ell M),(i, t+(\ell+1) M)]}$ and $s_{-}=s_{[(i, t-M),(i, t))}$ with

$$
\left|g_{J}\left(s_{-}, s_{+}\right)\right| \leq C e^{-\frac{\gamma}{M}|J|}
$$

Since this last Proposition is rather standard in statistical mechanics, we defer its proof to the Appendix. For the proof of the other Propositions, we need some Lemmas.

Lemma 3 There exists $B<\infty$ such that $\psi_{\Lambda \mathbf{s}}$, given by (11), satisfies

$$
\left|\psi_{\Lambda \mathbf{s}}(\mathbf{x})_{i}-\psi_{\Lambda^{\prime} \mathbf{s}^{\prime}}\left(\mathbf{x}^{\prime}\right)_{i}\right| \leq \lambda\left|x_{i}-x_{i}^{\prime}\right|+B \epsilon\left(\sum_{j \in \Lambda}\left|x_{j}-x_{j}^{\prime}\right| e^{-\beta|i-j|}+e^{-\beta d(i, \partial \Lambda)}\right)
$$

for $i \in \Lambda \subset \Lambda^{\prime}$, and $s=\left.s^{\prime}\right|_{\Lambda}$. For $\Lambda=\Lambda^{\prime}$, we get (31) without the term $e^{-\beta d(i, \partial \Lambda)}$.

Proof Proceeding as in $(5,6)$, and using Lemma 1 for $a_{\Lambda}^{-1}$, we get

$$
\left|a_{\Lambda}^{-1}(\mathbf{x})_{i}-a_{\Lambda^{\prime}}^{-1}\left(\mathbf{x}^{\prime}\right)_{i}\right| \leq\left|x_{i}-x_{i}^{\prime}\right|+B \epsilon\left(\sum_{j \in \Lambda}\left|x_{j}-x_{j}^{\prime}\right| e^{-\beta|i-j|}+e^{-\beta d(i, \partial \Lambda)}\right)
$$

Now, by (3.1), $\left(f^{-1}\right)^{\prime}<\lambda$ and this implies the claim.

Iterating (31) then yields 
Lemma 4 Let $\psi_{\mathbf{s}} \equiv \psi_{\Lambda \mathbf{s}}, \psi_{\mathbf{s}^{\prime}}^{\prime} \equiv \psi_{\Lambda^{\prime} \mathbf{s}^{\prime}}$. Then, there exist $C<\infty$ such that

$$
\begin{aligned}
& \left|\psi_{\mathbf{s}_{t_{n}}} \cdots \psi_{\mathbf{s}_{t_{1}}}(\mathbf{x})_{i}-\psi_{\mathbf{s}^{\prime} t_{n}}^{\prime} \cdots \psi_{\mathbf{s}^{\prime} t_{1}}^{\prime}\left(\mathbf{x}^{\prime}\right)_{i}\right| \\
& \leq \tilde{\lambda}^{n}\left(\left|x_{i}-x_{i}^{\prime}\right|+C \epsilon \sum_{j \in \Lambda} e^{-\frac{\beta}{2}|i-j|}\left|x_{j}-x_{j}^{\prime}\right|\right)+C \epsilon e^{-\frac{\beta}{2} d(i, \partial \Lambda)}
\end{aligned}
$$

where $i \in \Lambda \subset \Lambda^{\prime}, s=\left.s^{\prime}\right|_{\Lambda}$ and $\tilde{\lambda}=\lambda^{1 / 2}$. For $\Lambda=\Lambda^{\prime}$, we get (32) without the term $C \epsilon e^{-\frac{\beta}{2} d(i, \partial \Lambda)}$.

Proof of Lemma 4 The proof is done by induction. For $n=1$, we can use Lemma 3. Using (31) and (32) for $n-1$, we get

$$
\begin{aligned}
& \left|\psi_{\mathbf{s}_{t_{n}}} \cdots \psi_{\mathbf{s}_{t_{1}}}(\mathbf{x})_{i}-\psi_{\mathbf{s}^{\prime} t_{n}}^{\prime} \cdots \psi_{\mathbf{s}^{\prime} t_{1}}^{\prime}\left(\mathbf{x}^{\prime}\right)_{i}\right| \\
& \leq \lambda \tilde{\lambda}^{n-1}\left(\left|x_{i}-x_{i}^{\prime}\right|+C \epsilon \sum_{j \in \Lambda} e^{-\frac{\beta}{2}|i-j|}\left|x_{j}-x_{j}^{\prime}\right|\right)+C \epsilon \lambda e^{-\frac{\beta}{2} d(i, \partial \Lambda)} \\
& +B \epsilon\left[\sum_{j \in \Lambda} \tilde{\lambda}^{n-1}\left|x_{j}-x_{j}^{\prime}\right| e^{-\beta \mid i-j]}+C \epsilon \tilde{\lambda}^{n-1} \sum_{k, j \in \Lambda} e^{-\frac{\beta}{2}|j-k|}\left|x_{k}-x_{k}^{\prime}\right| e^{-\beta|i-j|}\right. \\
& \left.+C \epsilon \sum_{j \in \Lambda} e^{-\frac{\beta}{2} d(j, \partial \Lambda)} e^{-\beta|i-j|}\right]+B \epsilon e^{-\beta d(i, \partial \Lambda)}
\end{aligned}
$$

Regrouping terms, and exchanging $k, j$ in $\sum_{k, j}$, we get

$$
\begin{aligned}
& \leq \lambda \tilde{\lambda}^{n-1}\left|x_{i}-x_{i}^{\prime}\right| \\
& +\left(\lambda \tilde{\lambda}^{n-1} C \epsilon+B \epsilon \tilde{\lambda}^{n-1}+B C \epsilon^{2} \tilde{\lambda}^{n-1} \sum_{k \in \Lambda} e^{-\frac{\beta}{2}|i-k|}\right) \sum_{j \in \Lambda} e^{-\frac{\beta}{2}|i-j|}\left|x_{j}-x_{j}^{\prime}\right| \\
& +\left(C \epsilon \lambda+B C \epsilon^{2} \sum_{j \in \Lambda} e^{-\frac{\beta}{2}|i-j|}+B \epsilon\right) e^{-\frac{\beta}{2} d(i, \partial \Lambda)}
\end{aligned}
$$

Now choose $C$ large enough so that $\lambda C+\frac{B}{\lambda}+\mathcal{O}(\epsilon)<\tilde{\lambda} C$ (which is possible since $\left.\tilde{\lambda}=\lambda^{1 / 2}>\lambda\right)$ and we get (32) for $n$.

Remark It is easy to extend Lemmas 3 and 4 to the situation where $\Lambda=\Lambda^{\prime}$ but $s \neq s^{\prime}$ and to get, instead of (32),

$$
\begin{aligned}
& \quad \psi_{\mathbf{s}_{t_{n}}} \circ \cdots \circ \psi_{\mathbf{s}_{t_{1}}}(\mathbf{x})_{i}-\psi_{\mathbf{s}_{t_{n}}^{\prime}} \circ \cdots \circ \psi_{\mathbf{s}_{t_{1}}^{\prime}}\left(\mathbf{x}^{\prime}\right)_{i} \mid \\
& \leq \quad \tilde{\lambda}^{n}\left(\left|x_{i}-x_{i}^{\prime}\right|+C \epsilon \sum_{j \in \Lambda} e^{-\frac{\beta}{2}|i-j|}\left|x_{j}-x_{j}^{\prime}\right|\right) \\
& +\sum_{k=1}^{n} \tilde{\lambda}^{(n-k+1)}\left(\left|s_{\left(t_{k}, i\right)}-s_{\left(t_{k}, i\right)}^{\prime}\right|+C \epsilon \sum_{j \in \Lambda} e^{-\frac{\beta}{2}|i-j|}\left|s_{\left(t_{k}, j\right)}-s_{\left(t_{k}, j\right)}^{\prime}\right|\right)
\end{aligned}
$$

Proof of Proposition 1 Using (15) and (18) we can write

$$
\Phi_{Y_{\alpha}^{m}}^{\Lambda}(s)=g\left(\psi_{\overline{\mathbf{s}}_{t}} \circ \cdots \circ \psi_{\overline{\mathbf{s}}_{l}}(\mathbf{y})\right)-g\left(\psi_{\overline{\mathbf{s}}_{t}} \circ \cdots \circ \psi_{\overline{\mathbf{s}}_{l}}(\overline{\mathbf{y}})\right)
$$


where $\alpha=(t, i), l=t-t_{m}+1, \overline{\mathbf{s}}_{t_{k}}, t_{k}=t, \ldots, l$ is a configuration that coincides with $\mathbf{s}_{t_{k}}$ in $Y_{\alpha}^{m}$ and with 0 outside, $\mathbf{y}=\psi_{\overline{\mathbf{s}}_{t-t_{m}}} \circ \psi_{\mathbf{0}} \circ \cdots \circ \psi_{\mathbf{0}}(\mathbf{0})$ and $\overline{\mathbf{y}}$ is defined similarly, but with $\overline{\mathbf{s}}_{t-t_{m}}$ coinciding with $\mathbf{s}_{t-t_{m}}$ in $Y_{\alpha}^{m-1}$ and with 0 outside, so that $\bar{y}_{j}=y_{j}$ for $|i-j|<\left|i_{m}\right|$. Finally, $g$ is given by (15).

Using the Hölder continuity of $f^{\prime}$ and Lemma 2, we get

$$
\begin{aligned}
& \left|\Phi_{Y_{\alpha}^{m}}^{\Lambda}(s)\right| \leq C\left|\left(\psi_{\overline{\mathbf{s}}_{t}} \circ \cdots \circ \psi_{\overline{\mathbf{s}}_{l}}(\mathbf{y})\right)_{i}-\left(\psi_{\overline{\mathbf{s}}_{t}} \circ \cdots \circ \psi_{\overline{\mathbf{s}}_{l}}(\overline{\mathbf{y}})\right)_{i}\right|^{\delta} \\
& +C \epsilon \sum_{k \in \Lambda} e^{-\frac{\beta}{2}|i-k|} \mid\left(\psi_{\overline{\mathbf{s}}_{t}} \circ \cdots \circ \psi_{\overline{\mathbf{s}}_{l}}(\mathbf{y})\right)_{k}-\left(\left.\psi_{\overline{\mathbf{s}}_{t}} \circ \cdots \circ \psi_{\overline{\mathbf{s}}_{l}}(\overline{\mathbf{y}})_{k}\right|^{\delta}\right.
\end{aligned}
$$

Now, use (32) in Lemma 4 for $\Lambda=\Lambda^{\prime}$ and the fact that $\bar{y}_{j}=y_{j}$ for $|i-j|<\left|i_{m}\right|$ to get (23). By definition, $\tilde{\lambda}^{\delta}=\lambda^{\delta / 2}=\lambda^{\prime}$.

For (24), we bound

$$
\begin{aligned}
& \left|\Phi_{Y_{\alpha}^{m}}^{\Lambda}(s)-\Phi_{Y_{\alpha}^{m}}^{\Lambda^{\prime}}(s)\right| \\
& \leq\left|g\left(\psi_{\overline{\mathbf{s}}_{t}} \circ \cdots \circ \psi_{\overline{\mathbf{s}}_{l}}(\mathbf{y})\right)-g\left(\psi_{\overline{\mathbf{s}}_{t}^{\prime}}^{\prime} \circ \cdots \circ \psi_{\overline{\mathbf{s}}_{l}^{\prime}}^{\prime}(\mathbf{y})\right)\right| \\
& +\left|g\left(\psi_{\overline{\mathbf{s}}_{t}} \circ \cdots \circ \psi_{\overline{\mathbf{s}}_{l}}(\overline{\mathbf{y}})\right)-g\left(\psi_{{\overline{\mathbf{s}_{t}^{\prime}}}_{t}^{\prime}}^{\prime} \cdots \circ \psi_{\overline{\mathbf{s}}_{l}^{\prime}}^{\prime}(\overline{\mathbf{y}})\right)\right|
\end{aligned}
$$

where $\psi_{\overline{\mathbf{s}}^{\prime}}^{\prime}=\psi_{\Lambda^{\prime} \overline{\mathbf{s}}^{\prime}}$ and $\overline{\mathbf{s}}_{t}^{\prime}$ is defined like $\overline{\mathbf{s}}_{t}$, but with $\Lambda^{\prime}$ instead of $\Lambda$. Now use the Hölder continuity of $f^{\prime}$ and Lemma 2 and then, use (32), where only the last term contributes.

Proof of Proposition 2 The bounds (26), (27) follow from Proposition 1 because we excluded from $\Phi^{1}$ the terms with $\delta_{0 i_{m}}=0$ and $t_{m}<M$, and because of our choice of $M$ in (25): To get (26), we use $d(X) \leq C\left(t_{m}+\left|i_{m}\right|\right)$ which follows from (20) and from the definition of $Y_{\alpha}^{m}$.

Proof of Theorem 3 We shall construct $\mu$ as the weak limit of $T_{\Lambda}^{* N} m_{\Lambda}=\left(P_{\Lambda}^{N} 1\right) m_{\Lambda}$, as $N \rightarrow \infty, \Lambda \uparrow \mathbf{Z}^{d}$, and, for that, we shall use the results of Section 2, the representations $(13,21,22)$ and the bounds of Proposition 2. Thus, let $G \in L^{\infty}\left(\mathcal{M}_{B}\right),|B|<\infty$; we have

$$
\int G \circ T_{\Lambda}^{N} d m_{\Lambda}=\sum_{\left(\mathbf{s}_{i}\right)_{1}^{N}} \int_{C_{\Lambda}} G(\mathbf{x}) \exp \left(-\mathcal{H}_{\Lambda_{N}}(s)\right) d \mathbf{x}
$$

We shall first construct the unique Gibbs state $\bar{\mu}$ with Hamiltonian $\mathcal{H}$ on the following "mixed" phase space: the lattice is $\mathbf{Z}_{+}^{d+1}, \Omega_{\alpha}=S^{1}$ if $\alpha=(0, i), i \in \mathbf{Z}^{d}$ and $\Omega_{\alpha}=$ $\{0, \ldots, k-1\}$ if $\alpha=(t, i), t>0$. Then, $\mu$ will be the restriction of $\bar{\mu}$ to the "time zero" phase space $\mathcal{M}$, as shown by (34). Since we want to use Theorem 2.2 (or, rather a trivial extension of it to the present setting), let us check now that our system satisfies the hypotheses of this theorem. We cover $\mathbf{Z}_{+}^{d+1}$ with $L$-cubes, $L=\ell_{0} M$, where

$$
M e^{-\gamma \ell_{0}} \leq \epsilon^{\delta}
$$

(here, it would be more natural to take, instead of cubes, segments parallel to the time axis, but we shall keep the notations of Section 2). We bound $\left\|\Phi_{\Lambda}^{1}\right\|_{2} \leq \epsilon$ uniformly in $\Lambda$ 
and we get (2.33) from Proposition 2 (with $\epsilon=C \epsilon^{\delta}$ of Proposition 2). We get $(2.13,2.14$ ) from (28) and Proposition 3 as follows. Let $f=\log \lambda$ and $\phi_{Y}=\log \left(1+g_{J}\right)$ for $Y$ being and interval $J$ as in Proposition 3 with $|J|=n L, n \geq 1$ and $\phi_{Y}=0$ otherwise. Then $(30,35)$ imply $(2.14)$ (with $\epsilon$ replaced by $\left.C \epsilon^{\delta}\right)$, using

$$
\sum_{n \geq 1} L n e^{\frac{\gamma L n}{L}} e^{-\frac{\gamma L n}{M}}=M \sum_{n \geq 1} \ell_{0} n e^{-\gamma\left(\ell_{0}-1\right) n} \leq C M e^{-\gamma \ell_{0}}
$$

In order to be able to use estimates $(2.30,2.31)$ (see Remark 2.2 ), if we observe that $L=\ell_{0} M$ with $M$ given by (25) and $\ell_{0}$ by (35) so that $M \leq C|\log \epsilon|, \ell_{0} \leq C|\log \epsilon|$ and $L^{d+1} \epsilon^{\delta}<<1$ for $\epsilon$ small.

So, Theorem 2.2 proves the existence of $\mu$. Its invariance under $T$ and under the lattice translations follows by construction: Let $G \in \mathcal{C}\left(\mathcal{M}_{B}\right)$. Observe that since the limit $\Lambda \uparrow \mathbf{Z}^{d}, N \rightarrow \infty$ taken above can be taken in any order,

$$
\mu^{\Lambda}=\lim _{N \rightarrow \infty}\left(P_{\Lambda}^{N} 1\right) m_{\Lambda}
$$

exists and is $T_{\Lambda}$ invariant. On the other hand,

$$
\lim _{\Lambda \uparrow \mathbf{Z}^{d}}\left\|G \circ T_{\Lambda}-G \circ T\right\|_{\infty}=0
$$

since $G$ is continuous. Therefore,

$$
\begin{aligned}
& \int G \circ T d \mu=\lim _{\Lambda \uparrow \mathbf{Z}^{d}} \int G \circ T_{\Lambda} d \mu=\lim _{\Lambda \uparrow \mathbf{Z}^{d}} \lim _{\Lambda^{\prime} \uparrow \mathbf{Z}^{d}} \int G \circ T_{\Lambda} d \mu^{\Lambda^{\prime}} \\
& =\lim _{\Lambda \uparrow \mathbf{Z}^{d}} \lim _{\Lambda^{\prime} \uparrow \mathbf{Z}^{d}} \lim _{N \rightarrow \infty} \int G \circ T_{\Lambda} \circ T_{\Lambda^{\prime}}^{N} d m_{\Lambda^{\prime}}
\end{aligned}
$$

By (36), we may replace here $T_{\Lambda}$ by $T_{\Lambda^{\prime}}$ and we get

$$
(37)=\lim _{\Lambda^{\prime} \uparrow \mathbf{Z}^{d}} \lim _{N \rightarrow \infty} \int G \circ T_{\Lambda^{\prime}}^{N+1} d m_{\Lambda^{\prime}}=\int G d \mu
$$

The $\mathbf{Z}^{d}$-translation invariance of $\bar{\mu}$ implies the same invariance for $\mu$.

On the other hand, since the limit $\Lambda \uparrow \mathbf{Z}^{d}, N \rightarrow \infty$ of (34) exists for any $G \in$ $L^{\infty}\left(\mathcal{M}_{B}\right)$ with $|B|<\infty$, we see, by taking characteristic functions of sets of zero Lebesgue measure, that $\mu_{\Lambda}$ is absolutely continuous with respect to the Lebesgue measure, for any $|\Lambda|<\infty$.

The space-time exponential mixing of $\mu$ follows essentially from the exponential clustering of $\bar{\mu}$ which itself follows from Theorem 2.2: By an approximation argument, it is enough, to prove (3.10), to consider $G \in \mathcal{C}\left(\mathcal{M}_{B}\right)$, and $H$ as in (3.10): Then, as in (37),

$$
\begin{aligned}
& \int G \circ T^{n} H d \mu-\int G d \mu \int H d \mu \\
& =\lim _{\Lambda \uparrow \mathbf{Z}^{d}} \lim _{N \rightarrow \infty}\left(\int G P_{\Lambda}^{n}\left(H P_{\Lambda}^{N} 1\right) d m_{\Lambda}-\int G P_{\Lambda}^{N} 1 d m_{\Lambda} \int H P_{\Lambda}^{N} 1 d m_{\Lambda}\right) .
\end{aligned}
$$


Following equations $(10,12,13)$, we get (replacing $H$ by the periodic function $h)$ :

$$
P_{\Lambda}^{n}\left(h P^{N} 1\right)(\mathbf{x})=\sum_{\mathbf{s}_{1}, \cdots, \mathbf{s}_{N+n}} e^{-\mathcal{H}_{\Lambda_{N+n}}(s)} h(s)
$$

where

$$
h(s) \equiv h\left(\psi_{\mathbf{s}_{n}} \circ \cdots \circ \psi_{\mathbf{s}_{1}}(\mathbf{x})\right) .
$$

Proceeding as in (19), we write

$$
h(s)=\sum_{m \geq 0} h_{Y_{\alpha}^{m}}(s)+\text { constant }
$$

where $\alpha=(i, n)$ for some $i \in D$, and

$$
h_{Y_{\alpha}^{m}}(s)=h\left(s_{Y_{\alpha}^{m}} \vee 0_{\left(Y_{\alpha}^{m}\right)^{c}}\right)-h\left(s_{Y_{\alpha}^{m-1}} \vee 0_{\left(Y_{\alpha}^{m-1}\right)^{c}}\right)
$$

Since $h$ is Hölder continuous, proceeding as in the proof of Proposition 1, we get an estimate on $h_{Y_{\alpha}^{m}}$ similar to (23):

$$
\left|h_{Y_{\alpha}^{m}}(s)\right| \leq C\|H\|_{\delta} \lambda^{\prime t m} e^{-\beta^{\prime} d\left(i-i_{m}, D\right)}
$$

Now, insert $(39,41)$ in $(38)$, and use the exponential decay of correlation $(2.12)$ for $\bar{\mu}$ with $B$ being a subset of $\{0\} \times \mathbf{Z}^{d}$ and $A=Y_{\alpha}^{m}$, so that $d(A, B) \geq d\left(i-i_{m}, B\right)+\left(n-t_{m}\right)$, where the distance in the LHS is taken in $\mathbf{Z}_{+}^{d+1}$, while, on the RHS, it is taken in $\mathbf{Z}^{d}$. Then, from (42) and

$$
\sum_{\ell \geq 0} \exp \left(-m\left(d\left(i-i_{\ell}, B\right)+\left(n-t_{\ell}\right)\right)\right) \lambda^{\prime t_{\ell}} e^{-\beta^{\prime} d\left(i-i_{\ell}, D\right)} \leq C e^{-m^{\prime}(n+d(B, D))} \min (|B|,|D|)
$$

for some $m^{\prime}>0$, we get (3.10).

Finally, let $\nu$ be a regular state. We want to show that $\forall B \subset \mathbf{Z}^{d},|B|<\infty, \forall G \in$ $\mathcal{C}\left(\mathcal{M}_{B}\right)$

$$
\lim _{N \rightarrow \infty} \int G \circ T^{N} d \nu=\int G d \mu .
$$

Since $\left\|G \circ T_{\Lambda}^{N}-G \circ T^{N}\right\|_{\infty} \rightarrow 0$, as $\Lambda \uparrow \mathbf{Z}^{d}$, it is enough to show that

$$
\lim _{N \rightarrow \infty} \lim _{\Lambda \uparrow \mathbf{Z}^{d}}\left(\int G \circ T_{\Lambda}^{N} d \nu-\int G\left(P_{\Lambda}^{N} 1\right) d m_{\Lambda}\right)=0 .
$$

Since $G \circ T_{\Lambda}^{N}$ is $\mathcal{B}_{\Lambda}$ measurable we may replace $d \nu$ by $h_{\Lambda}\left(\mathbf{x}_{\Lambda}\right) d \mathbf{x}_{\Lambda}$ where $h_{\Lambda}=\frac{d \nu_{\Lambda}}{d m_{\Lambda}}$.

We have

$$
\begin{array}{r}
\int G \circ T_{\Lambda}^{N} h_{\Lambda} d m_{\Lambda}=\int_{C_{\Lambda}} g P_{\Lambda}^{N} h_{\Lambda} d \mathbf{x}_{\Lambda} \\
=\sum_{\left(\mathbf{s}_{i}\right)_{i=1}^{N}} \int_{C_{\Lambda}} g\left(\mathbf{x}_{B}\right) \exp \left(-\mathcal{H}_{\Lambda_{N}}(s)\right) h_{\Lambda}(s) d \mathbf{x}_{\Lambda}
\end{array}
$$


where $h_{\Lambda}(s)$ is defined by (40), with $n$ replaced by $N$. With the same notation, introduce $h_{\Lambda}(\bar{s})$ where

$$
\bar{s}_{\alpha}=0 \quad \forall \alpha=(t, i), \quad t \leq \frac{N}{2} \text { and } d(i, B) \leq N
$$

and $\bar{s}_{\alpha}=s_{\alpha}$ otherwise. Let

$$
\bar{h}_{\Lambda}(\bar{s})=\left(\sum_{\left(\mathbf{s}_{i}\right)_{i=1}^{N}} \int_{C_{\Lambda}} h_{\Lambda}(\bar{s}) e^{-\mathcal{H}_{\Lambda_{N}}(s)} d \mathbf{x}_{\Lambda}\right)^{-1} h_{\Lambda}(\bar{s})
$$

Insert the identity

$$
h_{\Lambda}(s)=\left(1-\frac{\bar{h}_{\Lambda}(\bar{s})}{h_{\Lambda}(s)}\right) h_{\Lambda}(s)+\bar{h}_{\Lambda}(\bar{s})
$$

in (44). Since

$$
\sum_{\left(\mathbf{s}_{i}\right)_{i=1}^{N}} \int_{C_{\Lambda}} h_{\Lambda}(s) e^{\mathcal{H}_{\Lambda_{N}}(s)} d \mathbf{x}_{\Lambda}=\int_{C_{\Lambda}} P_{\Lambda}^{N} h_{\Lambda} d \mathbf{x}_{\Lambda}=\int_{C_{\Lambda}} h_{\Lambda} d \mathbf{x}_{\Lambda}=1,
$$

(46) implies that $\frac{\bar{h}_{\Lambda}(\bar{s})}{h_{\Lambda}(s)}=\frac{h_{\Lambda}(\bar{s})}{h_{\Lambda}(s)}\left(\int \frac{h_{\Lambda}(\bar{s})}{h_{\Lambda}(s)} d \rho_{\Lambda}\right)^{-1}$, where $d \rho_{\Lambda}$ is a probability measure. So, we can bound

$$
\left|1-\frac{\bar{h}_{\Lambda}(\bar{s})}{h_{\Lambda}(s)}\right| \leq \exp \left(2 \sup _{s}\left|\log h_{\Lambda}(s)-\log h_{\Lambda}(\bar{s})\right|\right)-1 .
$$

Now, since $\nu$ is regular

$$
\left|\log h_{\Lambda}(s)-\log h_{\Lambda}(\bar{s})\right| \leq C \sum_{i}\left|\psi_{\mathbf{s}_{N}} \circ \cdots \circ \psi_{\mathbf{s}_{1}}(\mathbf{x})_{i}-\psi_{\overline{\mathbf{s}}_{N}} \circ \cdots \circ \psi_{\overline{\mathbf{s}}_{i}}(\overline{\mathbf{x}})_{i}\right|^{\delta}
$$

Now we use (33) and the definition (45) of $\bar{s}$ to bound each term in (50) by $C \lambda^{\mathcal{O}(N)}$ if $d(i, B) \leq N$ and by $C \lambda^{\mathcal{O}(N)} e^{-\frac{\delta \beta}{3}(d(i, B)-N)}$ if $d(i, B)>N$. Hence, (50) is bounded by $C(|B|) \lambda^{\mathcal{O}(N)}$. Combining this with (49), (47), (48) and since $G$ is bounded, we have

$$
\begin{aligned}
& \quad \int G \circ T_{\Lambda}^{N} d \nu-\int G\left(P_{\Lambda}^{N} 1\right) d m_{\Lambda} \mid \\
& \leq\left|\sum_{\left(\mathbf{s}_{i}\right)_{i=1}^{N}} \int_{C_{\Lambda}} g\left(\mathbf{x}_{B}\right) \exp \left(-\mathcal{H}_{\Lambda_{N}}(s)\right) \bar{h}_{\Lambda}(\bar{s}) d \mathbf{x}_{\Lambda}-\sum_{\left(\mathbf{s}_{i}\right)_{i=1}^{N}} \int_{C_{\Lambda}} g\left(\mathbf{x}_{B}\right) \exp \left(-\mathcal{H}_{\Lambda_{N}}(s)\right) d \mathbf{x}_{\Lambda}\right| \\
& +\quad C(|B|)\|G\|_{\infty} \lambda^{\mathcal{O}(N)}
\end{aligned}
$$

Since $g$ depends only on $\mathbf{x}_{B}$ and $\bar{h}_{\Lambda}(\bar{s})$ depends only on $s_{\alpha}$, with $\alpha=(t, i), t>\frac{N}{2}$ or $d(i, B)>N$ the first term in (51) is also bounded by $C G \lambda^{\mathcal{O}(N)}$, using the exponential decay of the Gibbs state defined by $\mathcal{H}$ (uniformly in $\Lambda$ and $N$ ) proven above and the normalization

$$
\sum_{\left(\mathbf{s}_{i}\right)_{i=1}^{N}} \int_{C_{\Lambda}} \exp \left(-\mathcal{H}_{\Lambda_{N}}(s)\right) \bar{h}_{\Lambda}(\bar{s}) d \mathbf{x}_{\Lambda}=1
$$


which follows from (46).

\section{Appendix}

Proof of Proposition 3 We need to study a one-dimensional system with potentials $\Phi_{Y}^{0}, Y \subset \mathbf{Z}_{+}, Y=\left[t_{1}, t_{1}+1, \cdots, t_{2}\right],\left|t_{2}-t_{1}\right|<M, t_{1} \geq 1$ satisfying

$$
\left|\Phi_{Y}^{0}\right| \leq C \lambda^{\prime|Y|}
$$

for $\lambda^{\prime}<1$ (we suppress here the $\Lambda$ dependence of $\Phi$ ).

Let $\mathcal{T}$ be the transfer matrix for $\Phi^{0}$, i.e. $\mathcal{T}$ is the linear operator on functions on $S \equiv\{0,1, \cdots, k-1\}^{M}$ (i.e. on $\mathbf{R}^{|S|}$ ) given by

$$
(\mathcal{T} f)(s)=\sum_{t \in S} \mathcal{T}(s, t) f(t)
$$

with

$$
\mathcal{T}(s, t)=a(s) a(t) e^{U(s, t)}
$$

where

$$
a(s)=\exp \left(\frac{1}{2} \sum_{Y \subset[1, M]} \Phi_{Y}^{0}(s)\right)
$$

and

$$
U(s, t)=\sum_{Y} \Phi_{Y}^{0}(s \vee t)
$$

where $Y \subset[1,2 M]$ with $M, M+1 \in Y$ and $(s \vee t)_{i}$; equals $s_{i}$ if $i \leq M$ and $t_{i-M}$ if $i>M$.

Let $J$ be as in the Proposition; we have

$$
Z^{0}(J \mid s)=\left(f_{s_{-}}, \mathcal{T}^{\ell} h_{s_{+}}\right)
$$

where $(\cdot, \cdot)$ is the scalar product in $\mathbf{R}^{|S|}$, and

$$
\begin{aligned}
& f_{s_{-}}(s)=a(s) e^{U\left(s_{-}, s\right)} \\
& h_{s_{+}}(t)=a(t) e^{U\left(t, s_{+}\right)}
\end{aligned}
$$

We can apply the Perron-Frobenius theory since $\mathcal{T}$ has strictly positive entries. $\mathcal{T}$ has a non-degenerate largest eigenvalue $\lambda$ with left and right eigenvectors $u$ and $v$ :

$$
\begin{aligned}
& u \mathcal{T}=\lambda u \\
& \mathcal{T} v=\lambda v
\end{aligned}
$$

The vectors $v$ and $u$ can be chosen with positive entries: $u(s), v(s)>0, \forall s \in S$ and we normalize them by

$$
\sum_{s \in S} u(s) v(s)=1>
$$

We may write $\mathcal{T}=\lambda Q+R$ where $Q(s, t)=v(s) u(t), Q^{2}=Q$ and $Q R=R Q=0$. 
Hence $\mathcal{T}^{\ell}=\lambda^{\ell} Q+R^{\ell}$, and

$$
\left(f_{s_{-}}, \mathcal{T}^{\ell} h_{s_{+}}\right)=\lambda^{\ell}\left(f_{s_{-}}, v\right)\left(u, h_{s_{+}}\right)\left(1+\frac{\left(f_{s_{-}}, R^{\ell} h_{s_{+}}\right)}{\lambda^{\ell}\left(f_{s_{-}}, v\right)\left(u, h_{s_{+}}\right)}\right)
$$

So define $W\left(s_{-}\right)=\left(f_{s_{-}}, v\right), W\left(s_{+}\right)=\left(u, h_{s_{+}}\right)$, and

$$
g_{J}\left(s_{-}, s_{+}\right)=\frac{\left(f_{s_{-}}, R^{\ell} h_{s_{+}}\right)}{\lambda^{\ell}\left(f_{s_{-}}, v\right)\left(u, h_{s_{+}}\right)}
$$

so that, using (A.6), $Z\left(J \mid s_{-}, s_{+}\right)$is of the form (4.29). To estimate $g_{J}$ it is convenient to introduce the matrix

$$
P(s, t)=\frac{\mathcal{T}(s, t) v(t)}{\lambda v(s)}
$$

Since $P(s, t)>0, \sum_{t} P(s, t)=1$, we can view $P(s, t)$ as the transition probability (from state $s$ to state $t$ ) of a Markov chain. $P$ has a unique stationary distribution $p P=p$, where $p(s)=u(s) v(s)$. Write

$$
P=\tilde{Q}+\tilde{R}
$$

where

$$
\tilde{Q}(s, t)=\frac{Q(s, t) v(t)}{v(s)}=u(t) v(t)=p(t)
$$

and

$$
\tilde{R}(s, t)=\frac{R(s, t) v(t)}{\lambda v(s)}
$$

We have $\tilde{Q} \tilde{R}=\tilde{R} \tilde{Q}=0$ and $P^{\ell}=\tilde{Q}+\tilde{R}^{\ell}$.

A standard result in the ergodic theory of Markov chains implies that

$$
\sum_{t}\left|\sum_{s} q(s) P^{\ell}(s, t)-p(t)\right| \leq 2(1-\gamma)^{\ell}
$$

where

$$
\gamma=\min _{s, s^{\prime}, t} \frac{P\left(s^{\prime}, t\right)}{P(s, t)}
$$

for any $q$, with $q(s) \geq 0, \sum_{s} q(s)=1$. Postposing the proof of (A.16), we rewrite it, using (A.13,A.14), as

$$
\sum_{t}\left|\sum_{s} q(s) \tilde{R}^{\ell}(s, t)\right| \leq 2(1-\gamma)^{\ell}
$$

We use (A.11,A.15) to write

$$
\begin{gathered}
\left|g_{J}\left(s_{-}, s_{+}\right)\right|=\left|\frac{\left(f_{s_{-}} v, \tilde{R}^{\ell} h_{s_{+}} v^{-1}\right)}{\left(f_{s_{-}}, v\right)\left(u, h_{s_{+}}\right)}\right| \\
\leq 2(1-\gamma)^{\ell} \frac{\max _{t} h_{s_{+}}(t) v(t)^{-1}}{\min _{s} h_{s_{+}}(s) v(s)^{-1}}
\end{gathered}
$$


using (A.18) with

$$
q(s)=\frac{f_{s_{-}}(s) v(s)}{\left(f_{s_{-}}, v\right)}=\frac{f_{s_{-}}(s) v(s)}{\sum_{t} f_{s_{-}}(t) v(t)},
$$

and using $\sum_{s} u(s) v(s)=1$ to bound $\left(u, h_{s_{+}}\right) \geq \min _{s} h_{s_{+}}(s) v(s)^{-1}$.

Now, we need to show that $\gamma>0$ uniformly in $M$ and we have to bound the last factor in (A.19) by a constant independent of $M$. Using (A.12,A.17)

$$
\gamma=\min _{s, s^{\prime}, t} \frac{\mathcal{T}\left(s^{\prime}, t\right) v(s)}{\mathcal{T}(s, t) v\left(s^{\prime}\right)}=\min _{s, s^{\prime}, t} \frac{\mathcal{T}\left(s^{\prime}, t\right) \sum_{r} \mathcal{T}(s, r) v(r)}{\mathcal{T}(s, t) \sum_{r} \mathcal{T}\left(s^{\prime}, r\right) v(r)}
$$

where we use (A.8) in the last equality. So, since $v(r)>0$,

$$
\gamma \geq \min _{s^{\prime}, t, s, r} \frac{\mathcal{T}\left(s^{\prime}, t\right) \mathcal{T}(s, r)}{\mathcal{T}(s, t) \mathcal{T}\left(s^{\prime}, r\right)} \geq e^{-4\|U\|}
$$

by (A.3). Using (A.1,A.5), we see that $\|U\| \leq C_{1}\left(\lambda^{\prime}\right)$, uniformly in $M$. Now consider

$$
\frac{h_{s_{+}}(t) v(s)}{h_{s_{+}}(s) v(t)} \leq \max _{s, t, r} \frac{h_{s_{+}}(t) \mathcal{T}(s, r)}{h_{s_{+}}(s) \mathcal{T}(t, r)} \leq e^{4\|U\|}
$$

by (A.3,A.7). Inserting (A.20) in (A.19) yields (4.30).

We are left with the proof of (A.16). Since $p P=p$ and since $\Sigma p(s)=\Sigma q(s)=1$, the LHS of (A.16) can be written as

$$
\sum_{t}\left|\sum_{s, s^{\prime}}\left(P^{\ell}(s, t)-P^{\ell}\left(s^{\prime}, t\right)\right) q(s) p\left(s^{\prime}\right)\right|,
$$

and it is enough to prove that

$$
\sum_{t}\left|P^{\ell}(s, t)-P^{\ell}\left(s^{\prime}, t\right)\right| \leq 2(1-\gamma)^{\ell}
$$

for all $s, s^{\prime}$. Let $f_{s, s^{\prime}}(t)=\operatorname{sign}\left(P^{\ell}(s, t)-P^{\ell}\left(s^{\prime}, t\right)\right)$. Then, the LHS of (A.21) equals

$$
\begin{gathered}
\left|\left(P^{\ell} f\right)(s)-\left(P^{\ell} f\right)\left(s^{\prime}\right)\right| \\
=\left|\sum_{z} P(s, z)\left(P^{\ell-1} f\right)(z)-P\left(s^{\prime}, z\right)\left(P^{\ell-1} f\right)(z)\right| \\
\leq \frac{1}{2}\left(\sum_{z}\left|P(s, z)-P\left(s^{\prime}, z\right)\right|\right) \sup _{z, z^{\prime}}\left|\left(P^{\ell-1} f\right)(z)-\left(P^{\ell-1} f\right)\left(z^{\prime}\right)\right|
\end{gathered}
$$

where, to get the last inequality, we use

$$
\left|\mu_{1}(F)-\mu_{2}(F)\right| \leq \frac{1}{2}\left(\sum_{z}\left|\mu_{1}(z)-\mu_{2}(z)\right|\right) \sup _{z, z^{\prime}}\left|F(z)-F\left(z^{\prime}\right)\right|
$$

(see e.g. [41 Lemma 5.1.8) where $\mu_{1}, \mu_{2}$ are the probability measures $\mu_{1}(z)=P(s, z)$, $\mu_{2}(z)=P\left(s^{\prime}, z\right)$ and $F=P^{\ell-1} f$. 
Since $P(s, z), P\left(s, z^{\prime}\right)$ are probability measures,

$$
\frac{1}{2} \sum_{z}\left|P(s, z)-P\left(s^{\prime}, z\right)\right|=\sum_{z}+\left(P(s, z)-P\left(s^{\prime}, z\right)\right)
$$

where the last sum runs over positive terms

$$
=\sum_{z}^{+}\left(1-\frac{P\left(s^{\prime}, z\right)}{P(s, z)}\right) P(s, z) \leq 1-\gamma
$$

by definition (A.17) of $\gamma$.

Inserting (A.23) in (A.22) and iterating yields (A.21), using in the last step

$$
\sup _{z, z^{\prime}}\left|f_{s, s^{\prime}}(z)-f_{s, s^{\prime}}\left(z^{\prime}\right)\right| \leq 2 .
$$

\section{Acknowledgments}

We would like to thank L. Bunimovich, A. van Enter, C. Maes and Y. Sinai for interesting discussions. This work was supported by NSF grant DMS-9205296, by EC grants SC1-CT91-0695 and CHRX-CT93-0411 and was done in part during a visit of the authors to the Mittag-Leffler Institute. 


\section{References}

[1] R. Bowen, Equilibrium states and the ergodic theory of Anosov diffeomorphisms, Lecture Notes in Mathematics, 470, Springer, New York (1975).

[2] J. Bricmont, A. Kupiainen, Phase transition in the 3d random field Ising model, Commun. Math. Phys. 116, 539-572 (1988).

[3] J. Bricmont, A. Kupiainen, Coupled analytic maps, to appear in Nonlinearity.

[4] D.C. Brydges, A short course on cluster expansions, in: Critical Phenomena, Random Systems, Gauge Theories. Les Houches Session XLIII, K. Osterwalder, R. Stora eds., Elsevier, p.139-183 (1984).

[5] L.A. Bunimovich, Y.G. Sinai, Space-time chaos in coupled map lattices, Nonlinearity, 1, 491-516 (1988).

[6] L.A. Bunimovich, Y.G. Sinai, Statistical mechanics of coupled map lattices, in Ref 229.

[7] L.A. Bunimovich, Coupled map lattices: One step forward and two steps back, preprint (1993), to appear in the Proceedings of the "Gran Finale" on Chaos, Order and Patterns, Como (1993).

[8] K.M. Campbell, D.A. Rand, A natural spatio-temporal measure for axiom A weakly coupled map lattices, preprint, Univ. of Warwick (1994).

[9] M. Cassandro, E. Olivieri, Renormalization group and analyticity in one dimension: a proof of Dobrushin's theorem, Commun. Math. Phys. 80, 255 (1981).

[10] R.L. Dobrushin, Gibbsian random fields for lattice systems with pairwise interactions, Funct. Anal. Appl. 2, 292-301 (1968).

[11] R.L. Dobrushin, The description of a random field by means of conditional probabilities and conditions on its regularity, Theory Prob. Appl. 13, 197-224 (1968).

[12] R.L. Dobrushin, M.R. Martirosyan, Nonfinite perturbations of the random Gibbs fields, Theor. Math. Phys. 74, 10-20 (1988).

[13] R.L. Dobrushin, M.R. Martirosyan, Possibility of high-temperature phase transitions due to the many particle nature of the potential, Theor. Math. Phys. 75, 443-448 (1988).

[14] R.L. Dobrushin, E.A. Pecherski, Uniqueness conditions for finitely dependent random fields, in Random Fields, 223-261, Vol.1, J. Fritz et al. eds, North-Holland, Amsterdam (1981).

[15] R.L. Dobrushin, S.B. Shlosman, Completely analytical Gibbs fields, in Statistical Physics and Dynamical Systems (Rigorous Results), 371-403, Birkäuser, Boston (1985). 
[16] R.L. Dobrushin, S.B. Shlosman, Completely analytical interactions: a constructive description, J. Stat. Phys. 46, 983-1014(1987).

[17] H. von Dreifus, A. Klein, J.F. Perez, Taming Griffiths' singularities: infinite differentiability of quenched correlation functions.

[18] A.C.D. van Enter, R. Fernandez, A remark on different norms and analyticity for many particle interactions, J. Stat. Phys., 56, 965-972 (1989).

[19] A.C.D van Enter, R. Fernandez, A. Sokal, Regularity properties and pathologies of position-space renormalization-group transformations, J. Stat. Phys., 72, 879-1167 (1993).

[20] M. Fisher, Critical temperatures for anisotropic Ising lattices. II. General upper bounds, Phys. Rev. 162, 480-485 (1967).

[21] G. Gallavotti, S. Miracle-Sole, Correlation functions of a lattice system, Commun. Math. Phys. 7, 274-288 (1968).

[22] R. B. Griffiths, Non-analytic behavior above the critical point in a random Ising ferromagnet, Phys. Rev. Lett. 23, 17-19 (1969).

[23] L. Gross, Decay of correlations in classical lattice models at high temperature, Commun. Math. Phys. 68, 9-27 (1979).

[24] L. Gross, Absence of second-order phase transitions in the Dobrushin uniqueness theorem, J. Stat. Phys. 25, 57-72 (1981).

[25] V.M. Gundlach, D.A. Rand, Spatial-temporal chaos: 1. Hyperbolicity, structural stability, spatial-temporal shadowing and symbolic dynamics, Nonlinearity 6, 165200 (1993); Spatial-temporal chaos: 2. Unique Gibbs states for higher-dimensional symbolic systems, Nonlinearity 6, 201-214 (1993); Spatial-temporal chaos: 3. Natural spatial-temporal measures for coupled circle map lattices, Nonlinearity 6, 215230 (1993).

[26] R.B. Israel, High-temperature analyticity in classical lattice systems, Commun. Math. Phys. 50, 245-257 (1976).

[27] M. Jiang, Equilibrium states for lattice models of hyperbolic type, to appear in Nonlinearity.

[28] M. Jiang, A. Mazel, Uniqueness of Gibbs states and exponential decay of correlations for some lattice models, preprint.

[29] K. Kaneko (ed): Theory and Applications of Coupled Map Lattices, J. Wiley (1993).

[30] K. Kaneko (ed): Focus Issue on Coupled Map Lattices, Chaos 2 (1993).

[31] G. Keller, M. Künzle, Transfer operators for coupled map lattices, Erg. Th. and Dyn. Syst. 12, 297-318 (1992). 
[32] V.A. Malyshev, R.A. Minlos, Gibbs Random Fields. Cluster Expansions, Kluwer, Dordrecht (1991).

[33] J. Miller, D.A. Huse, Macroscopic equilibrium from microscopic irreversibility in a chaotic coupled-map lattice, Phys. Rev. E, 48, 2528-2535 (1993).

[34] E. Olivieri, On a cluster expansion for lattice spin systems: a finite-size condition for the convergence, J. Stat. Phys., 50, 1179-1200 (1988).

[35] E. Olivieri, P. Picco, Cluster expansion for d-dimensional lattice systems and finite volume factorization properties, J. Stat. Phys., 59, 221-256 (1990).

[36] Y.G. Pesin, Y.G. Sinai, Space-time chaos in chains of weakly coupled hyperbolic maps, in: Advances in Soviet Mathematics, Vol. 3, ed. Y.G. Sinai, Harwood (1991).

[37] Y. Pomeau, Periodic behaviour of cellular automata, J. Stat. Phys. 70, 1379-1382 (1993).

[38] D. Ruelle, Thermodynamic Formalism, Addison-Wesley (1978).

[39] B. Simon, A remark on Dobrushin's uniqueness theorem, Commun. Math. Phys. 68, 183-185 (1979).

[40] B. Simon, The Statistical Mechanics of Lattice Gases, Vol. 1, Princeton Univ. Press (1994).

[41] Y.G. Sinai, Gibbs measures in ergodic theory, Russian Math. Surveys 27, 21-64 (1972).

[42] Y.G. Sinai, Topics in ergodic theory, Princeton University Press (1994).

[43] R. Temam, Infinite-Dimensional Dynamical Systems in Mechanics and Physics, Springer, New York, 1988.

[44] D.L. Volevich, Kinetics of coupled map lattices, Nonlinearity 4, 37-45 (1991).

[45] D.L. Volevich, The Sinai -Bowen-Ruelle measure for a multidimensional lattice of interacting hyperbolic mappings, Russ. Acad. Dokl. Math. 47, 117-121 (1993).

[46] D.L. Volevich, Construction of an analogue of Bowen-Ruelle-Sinai measure for a multidimensional lattice of interacting hyperbolic mappings, Russ. Acad. Math. Sbornik 79, 347-363 (1994). 